\title{
UNIPOTENT ALMOST CHARACTERS OF SIMPLE $p$-ADIC GROUPS, II
}

\author{
G. LuszTiG \\ Dedicated to Professor E. B. Dynkin for his 90th birthday
}

0.1. For any finite group $\Gamma$, a "nonabelian Fourier transform matrix" was introduced in [L1]. This is a square matrix whose rows and columns are indexed by pairs formed by an element of $\Gamma$ and an irreducible representation of the centralizer of that element (both defined up to conjugation). As shown in [L2], this matrix, which is unitary with square 1 , enters (for suitable $\Gamma$ ) in the character formulas for unipotent representations of a finite reductive group.

In this paper we extend the definition of the matrix above to the case where $\Gamma$ is a reductive group over $\mathbf{C}$. We expect that this new matrix (for suitable $\Gamma$ ) relates the characters of unipotent representations of a simple $p$-adic group and the unipotent almost characters of that simple $p$-adic group [L6]. The new matrix is defined in $\S 1$. The definition depends on some finiteness results established in 1.2. Several examples are given in 1.4-1.6 and 1.12. In $\S 2$ a conjectural relation with unipotent characters of $p$-adic groups is stated. In $\S 3$ we consider an example arising from an odd spin group which provides some evidence for the conjecture. Since the group $\Gamma$ which enters in the conjecture is described in the literature only up to isogeny, we give a more precise description for it (or at least for the derived subgroup of its identity component) in the Appendix.

Notation. If $G$ is an affine algebraic group and $g \in G$ we denote by $g_{s}$ (resp. $g_{u}$ ) the semisimple (resp. unipotent) part of $g$. Let $\mathcal{Z}_{G}$ be the centre of $G$ and let $G^{0}$ be the identity component of $G$. Let $G_{d e r}$ be the derived subgroup of $G$. If $g \in G$ and $G^{\prime}$ is a subgroup of $G$ we set $Z_{G^{\prime}}(g)=\left\{x \in G^{\prime} ; x g=g x\right\}$.

\section{A PAIRING}

1.1. Let $H$ be a reductive (but not necessarily connected) group over $\mathbf{C}$; let $\Sigma$ be the set of semisimple elements in $H$. Let $[H]$ be the set of irreducible components of $H$. For $x, y$ in $\Sigma$ we set $A_{x, y}=\left\{z \in H ; z x z^{-1} y=y z x z^{-1}\right\}$; we have $A_{x, y}=\sqcup_{h \in[H]} A_{x, y}^{h}$ where $A_{x, y}^{h}=A_{x, y} \cap h$. Now $Z_{H}^{0}(x) \times Z_{H}^{0}(y)$ acts on $A_{x, y}$ by $\left(v, v^{\prime}\right): z \mapsto v^{\prime} z v^{-1}$, leaving stable each of the subsets $A_{x, y}^{h}(h \in[H])$.

Supported in part by National Science Foundation grant DMS-0758262.

Typeset by $\mathcal{A} \mathcal{M} \mathcal{S}-\mathrm{T}_{\mathrm{E}} \mathrm{X}$ 
Lemma 1.2. The $Z_{H}^{0}(x) \times Z_{H}^{0}(y)$ action on $A_{x, y}$ in 1.1 has only finitely many orbits.

For any $c \in Z_{H}(y) \cap \Sigma$ we choose a maximal torus $T_{c}$ of $Z_{Z_{H}(y)}(c)$ and a maximal torus $T_{c}^{\prime}$ of $Z_{H}(c)$ that contains $T_{c}$. (Note that $T_{c} \subset Z_{H}(c)$.) We show:

(a) If $E$ is any semisimple $H$-conjugacy class in $H$ then $E \cap c T_{c}$ is finite.

Since $E$ is a finite union of $H^{0}$-conjugacy classes, it is enough to show that, if $E^{\prime}$ is any semisimple $H^{0}$-conjugacy class in $H$, then $E^{\prime} \cap c T_{c}$ is finite. By [L5, I, 1.14], $E^{\prime} \cap c T_{c}^{\prime}$ is finite. Since $T_{c} \subset T_{c}^{\prime}$, we see that $E^{\prime} \cap c T_{c}$ is also finite, as required.

Let $\left\{c_{i} ; i \in[1, n]\right\}$ be a collection of elements of $Z_{H}(y) \cap \Sigma$, one in each connected component of $Z_{H}(y)$. Let $\left\{c_{k}^{\prime} ; k \in\left[1, n^{\prime}\right]\right\}$ be a collection of elements of $Z_{H}(x) \cap \Sigma$, one in each connected component of $Z_{H}(x)$. Let $E$ be the $H$-conjugacy class of $x$. By (a), $E_{i}:=E \cap c_{i} T_{c_{i}}$ is a finite set for $i \in[1, n]$. For any $i \in[1, n], e \in E_{i}$ we set $P_{i, e}=\left\{g \in H ; g x g^{-1}=e\right\}$. We have $P_{i, e}=p_{i, e} Z_{H}(x)$ for some $p_{i, e} \in H$ hence

$$
P_{i, e}=\cup_{k \in\left[1, n^{\prime}\right]} p_{i, e} c_{k}^{\prime} Z_{H}^{0}(x) .
$$

Let $z \in A_{x, y}$. We have $z x z^{-1} \in Z_{H}(y) \cap \Sigma$ hence $z x z^{-1} \in c_{i} Z_{H}^{0}(y)$ for a unique $i \in[1, n]$. Using [L5, I, 1.14(c)] (with $G$ replaced by the reductive group $Z_{H}(y)$ ) we see that $z x z^{-1}$ is $Z_{H}^{0}(y)$-conjugate to an element of $c_{i} T_{c_{i}}$. Thus, $v^{\prime} z x z^{-1} v^{\prime-1} \in$ $c_{i} T_{c_{i}}$ for some $v^{\prime} \in Z_{H}^{0}(y)$. Hence $v^{\prime} z x z^{-1} v^{\prime-1}=e \in E_{i}$ so that $v^{\prime} z \in P_{i, e}$. Using (b) we see that $v^{\prime} z=p_{i, e} c_{k}^{\prime} v$ for some $v \in Z_{H}^{0}(x)$ and some $k \in\left[1, n^{\prime}\right]$. Hence $v^{\prime} z v^{-1}=p_{i, e} c_{k}^{\prime}$. We see that the finitely many elements $p_{i, e} c_{k}^{\prime}(i \in[1, n], k \in$ $\left.\left[1, n^{\prime}\right], e \in E_{i}\right)$ represent all the orbits in the lemma. This completes the proof.

Remark. The following result can be deduced from the lemma above.

(c) Let $C, C^{\prime}$ be two semisimple $H^{0}$-conjugacy classes in $H$. Let $X=\left\{\left(g, g^{\prime}\right) \in\right.$ $\left.C \times C^{\prime} ; g g^{\prime}=g^{\prime} g\right\}$. Then the $H^{0}$-action on $X, g_{1}:\left(g, g^{\prime}\right) \mapsto\left(g_{1} g g_{1}^{-1}, g_{1} g^{\prime} g_{1}^{-1}\right)$ has only finitely many orbits.

Let $x \in C, y \in C^{\prime}$. Let $\mathfrak{Z}$ be a set of representatives for the orbits of $Z_{H^{0}}(x) \times$ $Z_{H^{0}}(y)$ on $A_{x, y}^{H^{0}}$. This is a finite set. (Even the orbits of the smaller group $Z_{H}^{0}(x) \times$ $Z_{H}^{0}(y)$ form a finite set.) Let $\mathcal{O}$ be an $H^{0}$-orbit in $X$. In $\mathcal{O}$ we can find an element of the form $\left(z x z^{-1}, y\right)$ where $z \in H^{0}, z x z^{-1} y=y z x z^{-1}$ hence $z \in A_{x, y}^{H^{0}}$. Thus $z=v^{\prime} \zeta v^{-1}$ where $v^{\prime} \in Z_{H^{0}}(y), v \in Z_{H^{0}}(x), \zeta \in \mathfrak{Z}$. We have $\left(z x z^{-1}, y\right)=$ $\left(v^{\prime} \zeta v^{-1} x v \zeta^{-1} v^{\prime-1}, y\right)=\left(v^{\prime} \zeta x \zeta^{-1} v^{\prime-1}, y\right) \in \mathcal{O}$ hence $\left(\zeta x \zeta^{-1}, y\right) \in \mathcal{O}$. Thus the number of $H^{0}$-orbits in $X$ is $\leq|\mathfrak{Z}|$, proving (c).

1.3. In the remainder of this section we fix a (not necessarily connected) reductive group $H$ over $\mathbf{C}$ and a finite subgroup $\Lambda$ of $\mathcal{Z}_{H}$. Let $\Sigma$ be the set of semisimple elements in $H$.

A pair $x^{\prime}, y^{\prime}$ of commuting elements in $\Sigma$ is said to be adapted if there exists a maximal torus $\mathcal{T}$ of $H^{0}$ such that $x^{\prime} \mathcal{T} x^{\prime-1}=\mathcal{T}, y^{\prime} \mathcal{T} y^{\prime-1}=\mathcal{T},\left(\mathcal{T} \cap Z_{H}\left(x^{\prime}\right)\right)^{0}$ is a maximal torus of $Z_{H}\left(x^{\prime}\right)^{0}$ and $\left(\mathcal{T} \cap Z_{H}\left(y^{\prime}\right)\right)^{0}$ is a maximal torus of $Z_{H}\left(y^{\prime}\right)^{0}$. For example, if $x^{\prime}, y^{\prime}$ are contained in $H^{0}$ then $x^{\prime}, y^{\prime}$ is adapted if and only there exist a maximal torus of $H^{0}$ that contains $x^{\prime}$ and $y^{\prime}$; this condition is automatically satisfied if $\left(H^{0}\right)_{d e r}$ is simply connected. 
As in 1.1 , let $[H]$ be the set of connected components of $H$. Let $x, y \in \Sigma$ and $h \in[H]$.

For any $x \in \Sigma$ we set $\bar{Z}(x)=Z_{H}(x) /\left(Z_{H}^{0}(x) \Lambda\right)$. For $x \in \Sigma$ let $I_{x}$ be the set of isomorphism classes of irreducible representations $\sigma$ (over $\mathbf{C}$ ) of $Z_{H}(x)$ on which the subgroup $Z_{H}(x)^{0}$ acts trivially. Let $\hat{\Lambda}=\operatorname{Hom}\left(\Lambda, \mathbf{C}^{*}\right)$. If $x \in \Sigma$ and $\chi \in \hat{\lambda}$ we denote by $I_{x}^{\chi}$ the set of $\sigma \in I_{x}$ such that $\Lambda$ acts on $\sigma$ through $\chi$ times identity. Let $\tilde{\Sigma}$ be the set of pairs $(x, \sigma)$ where $x \in \Sigma$ and $\sigma \in I_{x}$. Now $H$ acts on $\tilde{\Sigma}$ by $f:(x, \sigma) \mapsto{ }^{f}(x, \sigma):=\left(f x f^{-1},{ }^{f} \sigma\right)$ where ${ }^{f} \sigma \in I_{f x f^{-1}}$ is obtained from $\sigma$ via $\operatorname{Ad}(f): Z_{H}(x) \stackrel{\sim}{\longrightarrow} Z_{H}\left(f x f^{-1}\right)$.

For any $\chi \in \hat{\Lambda}$ let $\tilde{\Sigma} \chi=\left\{(x, \sigma) \in \tilde{\Sigma} ; \sigma \in I_{x}^{\chi}\right\}$.

We assume given a function $\kappa: \Sigma \times \Sigma \times[H] \rightarrow \mathbf{C}$ such that

$\kappa\left(f x f^{-1}, f^{\prime} y f^{\prime-1}, f^{\prime} h f^{-1}\right)=\kappa(x, y, h)$

$\kappa(x, y, h)=\kappa\left(y, x, h^{-1}\right)$ for any

$\kappa\left(\zeta x, \zeta^{\prime} y, h\right)=\kappa(x, y, h)$

for any $(x, y, h) \in \Sigma \times \Sigma \times[H], f, f^{\prime} \in H, \zeta, \zeta^{\prime} \in \Lambda$.

We say that $\kappa$ is a weight function. For $(x, y, h) \in \Sigma \times \Sigma \times[H]$ let ${ }^{0} A_{x, y}^{h}$ be a set of representatives for the orbits of the action $\left(v, v^{\prime}\right): z \mapsto v^{\prime} z v^{-1}$ of $Z_{H}^{0}(x) \times Z_{H}^{0}(y)$ on $A_{x, y}^{h}$; this is a finite set, by 1.2 . Let ${ }^{0} \tilde{A}_{x, y}^{h}$ be the set of all $z \in{ }^{0} A_{x, y}^{h}$ such that $z x z^{-1}, y$ form an adapted pair for $H$.

We say that $\kappa$ is the standard weight function if $\kappa(x, y, h)=\left|{ }^{0} \tilde{A}_{x, y}^{h}\right|^{-1}$ whenever $\tilde{A}_{x, y}^{h} \neq \emptyset$ and $\kappa(x, y, h)=0$ whenever $\tilde{A}_{x, y}^{h}=\emptyset$. This weight function is clearly independent of the choice of ${ }^{0} A_{x, y}^{h}$.

We return to the general case. Let $(x, \sigma) \in \tilde{\Sigma},(y, \tau) \in \tilde{\Sigma}$. We set

$$
\begin{aligned}
& ((x, \sigma),(y, \tau))=\left|\Lambda /\left(\Lambda \cap H^{0}\right)\right|^{-1}|\bar{Z}(x)|^{-1}|\bar{Z}(y)|^{-1} \\
& \times \sum_{h \in[H]} \kappa(x, y, h) \sum_{z \in \in^{0} A_{x, y}^{h}} \overline{\operatorname{tr}\left(z x z^{-1}, \tau\right)} \operatorname{tr}\left(z^{-1} y z, \sigma\right) .
\end{aligned}
$$

(Clearly, this is independent of the choice of ${ }^{0} A_{x, y}^{h}$.)

In the case where $H$ is finite, $\Lambda=\{1\}$ and $\kappa$ is the standard weight function (which in this case satisfies $\kappa(x, y, h)=1$ whenever $A_{x, y}^{h} \neq 0$ ) this reduces to the pairing introduced in [L1].

Note that we can take ${ }^{0} A_{y, x}^{h}$ to be the image of ${ }^{0} A_{x, y}^{h^{-1}}$ under $z \mapsto z^{-1}$; it follows that $((y, \tau),(x, \sigma))=\overline{((x, \sigma),(y, \tau))}$. If $(x, \sigma) \in \tilde{\Sigma} \chi,(y, \tau) \in \tilde{\Sigma}^{\chi^{\prime}}$ with $\chi, \chi^{\prime} \in \hat{\Lambda}$ and if $\zeta, \zeta^{\prime} \in \Lambda$ then

$$
\left((\zeta x, \sigma),\left(\zeta^{\prime} y, \tau\right)\right)=\chi\left(\zeta^{\prime}\right) \overline{\chi^{\prime}(\zeta)}((x, \sigma),(y, \tau))
$$

(We can take ${ }^{0} A_{\zeta x, \zeta^{\prime} y}^{h}={ }^{0} A_{x, y}^{h}$. For $f, f^{\prime} \in H$ we have

$$
\left({ }^{f}(x, \sigma),{ }^{\prime}(y, \tau)\right)=((x, \sigma),(y, \tau))
$$


(We can take $\left.{ }^{0} A_{f x f^{-1}, f^{\prime} y f^{\prime-1}}^{h}=f^{\prime}\left({ }^{0} A_{x, y}^{f^{\prime-1} h f}\right) f^{-1}\right)$.

Now let $\mathbf{V}$ be the $\mathbf{C}$-vector space with basis consisting of the elements $(x, \sigma) \in \tilde{\Sigma}$. Then (a) extends to a form $():, \mathbf{V} \times \mathbf{V} \rightarrow \mathbf{C}$ which is linear in the first variable, antilinear in the second variable and satisfies $\left(v^{\prime}, v\right)=\overline{\left(v, v^{\prime}\right)}$ for $v, v^{\prime} \in \mathbf{V}$. We have $\mathbf{V}=\oplus_{\chi \in \hat{\Lambda}} \mathbf{V}^{\chi}$ where $\mathbf{V}^{\chi}$ is the subspace of $\mathbf{V}$ spanned by $(x, \sigma) \in \tilde{\Sigma}^{\chi}$. For any $\chi, \chi^{\prime}$ in $\hat{\Lambda}$ let

$$
\mathcal{J}_{\chi^{\prime}}^{\chi}=\left\{v \in \mathbf{V}^{\chi} ;\left(v, \mathbf{V}^{\chi^{\prime}}\right)=0\right\}, \quad \overline{\mathbf{V}}_{\chi^{\prime}}^{\chi}=\mathbf{V}^{\chi} / \mathcal{J}_{\chi^{\prime}}^{\chi}
$$

Then (, ) induces a pairing $\overline{\mathbf{V}}_{\chi^{\prime}}^{\chi} \times \overline{\mathbf{V}}_{\chi}^{\chi^{\prime}} \rightarrow \mathbf{C}$ (denoted again by $($,$) ) which is linear$ in the first variable, antilinear in the second variable and satisfies $\left(v_{1}^{\prime}, v_{1}\right)=\overline{\left(v_{1}, v_{1}^{\prime}\right)}$ for $v_{1}^{\prime} \in \overline{\mathbf{V}}_{\chi^{\prime}}^{\chi}, v_{1} \in \overline{\mathbf{V}}_{\chi}^{\chi^{\prime}}$. Let $\mathfrak{A}_{\chi^{\prime}}^{\chi}$ be the image of the $\tilde{\Sigma}^{\chi}$ under the obvious map $\mathbf{V}^{\chi} \rightarrow \overline{\mathbf{V}}_{\chi^{\prime}}^{\chi}$. When $\chi=1$ we denote by $(x, \sigma)_{\chi^{\prime}}$ the image of $(x, \sigma) \in \tilde{\Sigma}^{1}$ under $\mathbf{V}^{1} \rightarrow \overline{\mathbf{V}}_{\chi^{\prime}}^{1}$

1.4. In 1.4-1.6 we assume that $\kappa$ is the standard weight function. In this subsection we assume that $H=H^{0} \Lambda$ and that $\left(H^{0}\right)_{\text {der }}$ is simply connected. In this case any pair of commuting semisimple elements in $H$ is adapted. Let $x, y \in \Sigma$. We have $\bar{Z}(x)=\{1\}, \bar{Z}(y)=\{1\}, I_{x}^{1}=\{1\}, I_{y}^{1}=\{1\},|[H]|=\left|\Lambda /\left(\Lambda \cap H^{0}\right)\right|, A_{x, y}^{h} \neq \emptyset$ for any $h \in[H]$. From the definitions we see that $((x, 1),(y, 1))=1$. Hence $(x, 1)-\left(x^{\prime}, 1\right) \in \mathcal{J}_{1}^{1}$ for any $x, x^{\prime} \in \Sigma$. We see that $\mathfrak{A}_{1}^{1}$ consists of a single element $(1,1)_{1}$ which has inner product 1 with itself and $\operatorname{dim} \overline{\mathbf{V}}_{1}^{1}=1$. (This example applies to the situation in $1.7(\mathrm{a})$ with $u$ any unipotent element in $\mathcal{G}=S L_{N}(\mathbf{C})$.)

1.5. In this subsection we assume that $H=H^{0} \sqcup H^{1}$ and that $H^{0}$ is isomorphic to $\mathbf{C}^{*}$ (we denote by $\lambda \mapsto g_{\lambda}$ an isomorphism $\mathbf{C}^{*} \stackrel{\sim}{\longrightarrow} H^{0}$ ); we also assume that any $r \in H^{1}$ satisfies $r g_{\lambda} r^{-1}=g_{\lambda^{-1}}$ for all $\lambda \in \mathbf{C}^{*}$ and that $\Lambda=\mathcal{Z}_{H}$ that is, $\Lambda=\left\{1, g_{-1}\right\} \subset H^{0}$. In this case any pair of commuting semisimple elements in $H$ is adapted. We fix $r \in H^{1}$. We have $r^{2}=1$ or $r^{2}=g_{-1}$. The case where $r^{2}=1$ (resp. $r^{2}=g_{-1}$ ) arises in the situation in 1.7 (a) with $u$ a subregular unipotent element in $\mathcal{G}=\operatorname{Spin}_{2 n+1}(\mathbf{C})$ with $n$ even (resp. $n$ odd). We have

$$
\begin{gathered}
Z_{H}(1)=Z_{H}\left(g_{-1}\right)=H, Z_{H}\left(g_{\lambda}\right)=H^{0} \text { if } \lambda \in \mathbf{C}^{*}-\{1,-1\}, \\
Z_{H}(r)=\left\{1, g_{-1}, r, r g_{-1}\right\} .
\end{gathered}
$$

Hence

$$
|\bar{Z}(1)|=2,\left|\bar{Z}\left(g_{\lambda}\right)\right|=1 \text { if } \lambda \in \mathbf{C}^{*}-\{1,-1\},|\bar{Z}(r)|=2 .
$$

We have

$$
\begin{gathered}
A_{g_{\lambda}, g_{\lambda^{\prime}}}=H \text { for any } \lambda, \lambda^{\prime} \in \mathbf{C}^{*}, \quad A_{1, r}=H, \quad A_{g_{\lambda}, r}=\emptyset \text { for any } \lambda \in \mathbf{C}^{*}-\{1,-1\}, \\
A_{r, r}=\left\{1, g_{-1}, r, r g_{-1}, g_{i}, g_{-i}, r g_{i}, r g_{-i}\right\}, \text { where } i=\sqrt{-1}
\end{gathered}
$$


Hence we can take

$$
\begin{gathered}
{ }^{0} A_{g_{\lambda}, g_{\lambda^{\prime}}}^{H^{0}}=\{1\}, \quad{ }^{0} A_{g_{\lambda}, g_{\lambda^{\prime}}}^{H^{1}}=\{r\} \text { for any } \lambda, \lambda^{\prime} \in \mathbf{C}^{*}, \\
\qquad{ }^{0} A_{1, r}^{H^{0}}=\{1\}, \quad{ }^{0} A_{1, r}^{H^{1}}=\{r\} \\
{ }^{0} A_{g_{\lambda}, r}^{H^{0}}=\emptyset, \quad{ }^{0} A_{\lambda, r}^{H^{1}}=\emptyset \text { for any } \lambda \in \mathbf{C}^{*}-\{1,-1\}, \\
{ }^{0} A_{r, r}^{H^{0}}=\left\{1, g_{-1}, g_{i}, g_{-i}\right\}, \quad{ }^{0} A_{r, r}^{H^{1}}=\left\{r, r g_{-1}, r g_{i}, r g_{-i}\right\} .
\end{gathered}
$$

Let $\epsilon$ be the non-trivial 1-dimensional representation of $H$ which is trivial on $H^{0}$. The restriction of $\epsilon$ to $Z_{H}(r)$ is denoted again by $\epsilon$. From the previous results we can write the $5 \times 5$ matrix of inner products $((x, \sigma),(y, \tau))$ with $(x, \sigma)$ running through $(1,1),(1, \epsilon),(r, 1),(r, \epsilon),\left(g_{\lambda}, 1\right)$ (they index the rows), $(y, \tau)$ running through $(1,1),(1, \epsilon),(r, 1),(r, \epsilon),\left(g_{\lambda^{\prime}}, 1\right)$ (they index the columns) and with $\lambda, \lambda^{\prime} \in \mathbf{C}^{*}-\{1,-1\}:$

$$
\left(\begin{array}{ccccc}
\frac{1}{2} & \frac{1}{2} & \frac{1}{2} & \frac{1}{2} & 1 \\
\frac{1}{2} & \frac{1}{2} & -\frac{1}{2} & -\frac{1}{2} & 1 \\
\frac{1}{2} & -\frac{1}{2} & \frac{1}{2} & -\frac{1}{2} & 0 \\
\frac{1}{2} & -\frac{1}{2} & -\frac{1}{2} & \frac{1}{2} & 0 \\
1 & 1 & 0 & 0 & 2
\end{array}\right)
$$

From the results in 1.3 we see that $(1,1)_{1},(1, \epsilon)_{1},(r, 1)_{1},(r, \epsilon)_{1},\left(g_{\lambda}, 1\right)_{1}$ generate $\overline{\mathbf{V}}_{1}^{1}$ and from the matrix above we see that for any $\lambda \in \mathbf{C}^{*}-\{1,-1\}$ we have $\left(g_{\lambda}, 1\right)_{1}=(1,1)_{1}+(1, \epsilon)_{1}$. Hence $(1,1)_{1},(1, \epsilon)_{1},(r, 1)_{1},(r, \epsilon)_{1}$ generate $\overline{\mathbf{V}}_{1}^{1}$ and the matrix of their inner products is

$$
\left(\begin{array}{cccc}
\frac{1}{2} & \frac{1}{2} & \frac{1}{2} & \frac{1}{2} \\
\frac{1}{2} & \frac{1}{2} & -\frac{1}{2} & -\frac{1}{2} \\
\frac{1}{2} & -\frac{1}{2} & \frac{1}{2} & -\frac{1}{2} \\
\frac{1}{2} & -\frac{1}{2} & -\frac{1}{2} & \frac{1}{2}
\end{array}\right)
$$

which is nonsingular. We see that $\mathfrak{A}_{1}^{1}$ consists of $(1,1)_{1},(1, \epsilon)_{1},(r, 1)_{1},(r, \epsilon)_{1}$, $(1,1)_{1}+(1, \epsilon)_{1}$, and the first four of these elements form a basis of $\overline{\mathbf{V}}_{1}^{1}$.

1.6. Let $E$ be a vector space of dimension $2 n \geq 2$ over the field $F_{2}$ with two elements with a given nondegenerate symplectic form $\langle\rangle:, E \times E \rightarrow F_{2}$. In this subsection we assume that $H$ is a Heisenberg group attached to $\langle$,$\rangle that is, a finite$ group with a given surjective homomorphism $\psi: H \rightarrow E$ whose kernel consists of 1 and another central element $c$ with $c^{2}=1$ and in which for any $x, y \in E$ we have $\dot{x}^{2}=\dot{y}^{2}=c, \dot{x} \dot{y}=c^{\langle x, y\rangle} \dot{y} \dot{x}$ for any $\dot{x} \in \psi^{-1}(x), \dot{y} \in \psi^{-1}(y)$; we also assume that $\Lambda=\{1, c\}$. (This example arises in the situation in 1.7(a) with $u$ a suitable unipotent element in $\mathcal{G}$ of spin type.)

We have $Z_{H}(1)=Z_{H}(c)=H$ and for $x \in E-\{0\}, \dot{x} \in \psi^{-1}(x)$ we have $Z_{H}(x)=\psi^{-1}\left(\left\{x^{\prime} \in E ;\left\langle x, x^{\prime}\right\rangle=0\right\}\right)$. We denote the nontrivial character of $\Lambda$ 
by $\chi$. If $x \in E$ and $\dot{x} \in \psi^{-1}(x)$ then $I_{\dot{x}}^{1}$ can be identified with $E_{x}:=E / F_{2} x$ (to each $\bar{y} \in E_{x}$ corresponds the character $\xi \mapsto\langle\psi(\xi), y\rangle$ of $H$ where $y \in E$ represents $\bar{y}$ ). Now $I_{1}^{\chi}$ and $I_{c}^{\chi}$ consist of a single representation $\rho$ whose character is $2^{n}$ at $1,-2^{n}$ at $c$ and 0 at all other elements of $H$. If $x \in E-\{0\}$ and $\dot{x} \in \psi^{-1}(x)$ then $I_{\dot{x}}^{\chi}$ consists of two representations $\rho, \rho^{\prime}$ such that the character of $\rho$ at $1, c, \dot{x}, c \dot{x}$ is $2^{n-1},-2^{n-1}, 2^{n-1} i,-2^{n-1} i$ (respectively), the character of $\rho^{\prime}$ at $1, c, \dot{x}, c \dot{x}$ is $2^{n-1},-2^{n-1},-2^{n-1} i, 2^{n-1} i$ (respectively) and whose character at all other elements of $H$ is 0 ; here $i=\sqrt{-1}$. Let $x, x^{\prime}, y, y^{\prime} \in E$, let $\dot{x} \in \psi^{-1}(x)$, $\dot{x}^{\prime} \in \psi^{-1}\left(x^{\prime}\right)$ and let $\bar{y}$ (resp. $\left.\bar{y}^{\prime}\right)$ be the image of $y$ (resp. $\left.y^{\prime}\right)$ in $E_{x}$ (resp. $E_{x^{\prime}}$ ). From the definitions we have

$$
\begin{gathered}
\left((\dot{x}, \bar{y}),\left(\dot{x}^{\prime}, \bar{y}^{\prime}\right)\right)=0 \text { if }\left\langle x, x^{\prime}\right\rangle \neq 0 \\
\left((\dot{x}, \bar{y}),\left(\dot{x}^{\prime}, \bar{y}^{\prime}\right)\right)=2^{-2 n+2}(-1)^{\left\langle x^{\prime}, y\right\rangle+\left\langle x, y^{\prime}\right\rangle}\left(\delta_{x, 0}+1\right)^{-1}\left(\delta_{x^{\prime}, 0}+1\right)^{-1}
\end{gathered}
$$

if $\left\langle x, x^{\prime}\right\rangle=0$.

Let $x, x^{\prime}, y \in E$, let $\dot{x} \in \psi^{-1}(x), \dot{x}^{\prime} \in \psi^{-1}\left(x^{\prime}\right)$ and let $\bar{y}$ be the image of $y$ in $E_{x} ;$ let $\rho_{1} \in I_{\dot{x}^{\prime}}^{\chi}$. From the definitions we have

$$
\begin{gathered}
\left((\dot{x}, \bar{y}),\left(\dot{x}^{\prime}, \rho_{1}\right)\right)=0 \text { if } x \neq 0, \\
\left((\dot{x}, \bar{y}),\left(\dot{x}^{\prime}, \rho_{1}\right)\right)=2^{-n}(-1)^{\left\langle x^{\prime}, y\right\rangle} \text { if } \dot{x}=1, \\
\left((\dot{x}, \bar{y}),\left(\dot{x}^{\prime}, \rho_{1}\right)\right)=-2^{-n}(-1)^{\left\langle x^{\prime}, y\right\rangle} \text { if } \dot{x}=c .
\end{gathered}
$$

Let $x, x^{\prime} \in E$, let $\dot{x} \in \psi^{-1}(x), \dot{x}^{\prime} \in \psi^{-1}\left(x^{\prime}\right)$ and let $\rho_{1} \in I_{\dot{x}}^{\chi}, \rho_{1}^{\prime} \in I_{\dot{x}^{\prime}}^{\chi}$. From the definitions we see that $\left(\left(\dot{x}, \rho_{1}\right),\left(\dot{x}^{\prime}, \rho_{1}^{\prime}\right)\right)$ is equal 0 if $x \neq x^{\prime}$, is equal to 1 if $\dot{x}=\dot{x}^{\prime}, r_{1}=r_{1}^{\prime}$ or if $\dot{x}^{\prime}=c \dot{x}, r_{1} \neq r_{1}^{\prime}$ and is equal to -1 if $\dot{x}^{\prime}=c \dot{x}, r_{1}=r_{1}^{\prime}$ or if $\dot{x}^{\prime}=\dot{x}, r_{1} \neq r_{1}^{\prime}$.

Let $Z$ be the set of all pairs $(x, \bar{y})$ where $x \in E$ and $\bar{y} \in E_{x}$; let $\mathcal{M}$ be the matrix with rows and columns indexed by $Z$ with entries $\mathcal{M}_{(x, \bar{y}),\left(x^{\prime}, \bar{y}^{\prime}\right)}=\left((\dot{x}, \bar{y}),\left(\dot{x}^{\prime}, \bar{y}^{\prime}\right)\right)$ where $\dot{x} \in \psi^{-1}(x), \dot{x}^{\prime} \in \psi^{-1}\left(x^{\prime}\right)$. Its square $\mathcal{M}^{2}$ has entries

$$
\mathcal{M}_{(x, \bar{y}),\left(x^{\prime}, \bar{y}^{\prime}\right)}^{2}=\sum_{\left(x^{\prime \prime}, \bar{y}^{\prime \prime}\right) \in Z}\left((\dot{x}, \bar{y}),\left(\dot{x}^{\prime \prime}, \bar{y}^{\prime \prime}\right)\right)\left(\left(\dot{x}^{\prime \prime}, \bar{y}^{\prime \prime}\right),\left(\dot{x}^{\prime}, \bar{y}^{\prime}\right)\right)
$$

where $\dot{x} \in \psi^{-1}(x), \dot{x}^{\prime} \in \psi^{-1}\left(x^{\prime}\right), \dot{x}^{\prime \prime} \in \psi^{-1}\left(x^{\prime \prime}\right)$. Let $x, x^{\prime}, y, y^{\prime} \in E$ and let $\bar{y}$ (resp. 
$\left.\bar{y}^{\prime}\right)$ be the image of $y\left(\operatorname{resp} . y^{\prime}\right)$ in $E_{x}$ (resp. $\left.E_{x^{\prime}}\right)$. From the definitions we have

$$
\begin{aligned}
& \mathcal{M}_{(x, \bar{y}),\left(x^{\prime}, \bar{y}^{\prime}\right)}^{2}=2^{-4 n+4} \sum_{\left(x^{\prime \prime}, y^{\prime \prime}\right) \in E \times E ;\left\langle x, x^{\prime \prime}\right\rangle=0,\left\langle x^{\prime \prime}, x^{\prime}\right\rangle=0} \\
& (-1)^{\left\langle x^{\prime \prime}, y+y^{\prime}\right\rangle+\left\langle x+x^{\prime}, y^{\prime \prime}\right\rangle}\left(\delta_{x, 0}+1\right)^{-1}\left(\delta_{x^{\prime \prime}, 0}+1\right)^{-2}\left(2-\delta_{x^{\prime \prime}, 0}\right)^{-1}\left(\delta_{x^{\prime}, 0}+1\right)^{-1} \\
& =\delta_{x, x^{\prime}} 2^{-2 n+4} \sum_{x^{\prime \prime} \in E ;\left\langle x, x^{\prime \prime}\right\rangle=0}(-1)^{\left\langle x^{\prime \prime}, y+y^{\prime}\right\rangle}\left(\delta_{x, 0}+1\right)^{-2}\left(\delta_{x^{\prime \prime}, 0}+1\right)^{-1} 2^{-1} \\
& =\delta_{x, x^{\prime}} 2^{-2 n+3} \sum_{x^{\prime \prime} \in E ;\left\langle x, x^{\prime \prime}\right\rangle=0}(-1)^{\left\langle x^{\prime \prime}, y+y^{\prime}\right\rangle}\left(\delta_{x, 0}+1\right)^{-2} \\
& -\delta_{x, x^{\prime}} 2^{-2 n+3}\left(\delta_{x, 0}+1\right)^{-2}+\delta_{x, x^{\prime}} 2^{-2 n+3}\left(\delta_{x, 0}+1\right)^{-2} 2^{-1} \\
& =\delta_{x, x^{\prime}} \delta_{\bar{y}, \bar{y}^{\prime}} 2^{-2 n+3}\left|\left\{x^{\prime \prime} \in E ;\left\langle x, x^{\prime \prime}\right\rangle=0\right\}\right|\left(\delta_{x, 0}+1\right)^{-2} \\
& -\delta_{x, x^{\prime}} 2^{-2 n+2}\left(\delta_{x, 0}+1\right)^{-2} \\
& =\delta_{x, x^{\prime}} \delta_{\bar{y}, \bar{y}^{\prime}} 2^{-2 n+3} 2^{2 n-1}\left(\delta_{x, 0}+1\right)^{-1}-\delta_{x, x^{\prime}} 2^{-2 n+2}\left(\delta_{x, 0}+1\right)^{-2} \\
& =\delta_{x, x^{\prime}}\left(2-\delta_{x, 0}\right)\left(\delta_{\bar{y}, \bar{y}^{\prime}} 2-\left|E_{x}\right|^{-1}\right) .
\end{aligned}
$$

We see that $\mathcal{M}^{2}$ is a direct sum over $x \in E$ of matrices $\mathcal{P}(x)$ indexed by $E_{x} \times E_{x}$ where $\mathcal{P}(x)=\left(2-\delta_{x, 0}\right)\left(2 I-\left|E_{x}\right|^{-1} J\right)$ and all entries of $J$ are 1 . Thus we have $J^{2}=\left|E_{x}\right| J$. Setting $\mathcal{P}^{\prime}(x)=\left(2-\delta_{x, 0}\right)^{-1} \mathcal{P}(x)$ we have $\left|E_{x}\right|^{-1} J=2 I-\mathcal{P}^{\prime}(x)$ hence

$$
\mathcal{P}^{\prime}(x)^{2}-4 \mathcal{P}^{\prime}(x)+4 I=\left(2 I-\mathcal{P}^{\prime}(x)\right)^{2}=\left|E_{x}\right|^{-2} J^{2}=\left|E_{x}\right|^{-1} J=2 I-\mathcal{P}^{\prime}(x) .
$$

Thus $\mathcal{P}^{\prime}(x)^{2}-3 \mathcal{P}^{\prime}(x)+2 I=0$ that is $\left(\mathcal{P}^{\prime}(x)-I\right)\left(\mathcal{P}^{\prime}(x)-2 I\right)=0$. This shows that $\mathcal{P}^{\prime}(x)$ is semisimple with eigenvalues 1 and 2 hence $\mathcal{M}^{2}$ is semisimple with eigenvalues $2-\delta_{x, 0}$ and $2\left(2-\delta_{x, 0}\right)$ hence $\mathcal{M}$ is semisimple with eigenvalues $\pm 1, \pm \sqrt{2}, \pm 2$. In particular $\mathcal{M}$ is invertible.

For $z=(x, \bar{y}) \in Z$ we set $a_{z}=(\dot{x}, \bar{y})_{1} \in \overline{\mathbf{V}}_{1}^{1}$ (where $\left.\dot{x} \in \psi^{-1}(x)\right)$; this is independent of the choice of $\dot{x}$. Note that $\mathfrak{A}_{1}^{1}$ consists of the elements $a_{z}$. From the fact that $\mathcal{M}$ is invertible we see that $\left\{a_{z} ; z \in Z\right\}$ is a basis of $\overline{\mathbf{V}}_{1}^{1}$.

1.7. Let $\mathcal{G}$ be a connected, simply connected, almost simple reductive group over C. Let $u$ be a unipotent element of $\mathcal{G}$. Let $\mathcal{V}$ be the unipotent radical of $Z_{\mathcal{G}}(u)^{0}$.

(a) Until the end of 1.11 we assume that $H=H(u)$ is a reductive subgroup of $Z_{\mathcal{G}}(u)$ such that $Z_{\mathcal{G}}(u)=H \mathcal{V}, H \cap \mathcal{V}=\{1\}$ and that $\Lambda=\mathcal{Z}_{\mathcal{G}}$.

(It is well known that such $H$ exists and that $H$ is unique up to $Z_{\mathcal{G}}$-conjugacy; note that $\mathcal{Z}_{\mathcal{G}} \subset H$.) We can state the following result in which we allow $\kappa$ to be any weight function for $H$.

Proposition 1.8. Let $\chi, \chi^{\prime} \in \hat{\Lambda}$. Then $\mathfrak{A}_{\chi^{\prime}}^{\chi}$ is finite. In particular, $\operatorname{dim} \overline{\mathbf{V}}_{\chi^{\prime}}^{\chi}<\infty$.

The proof is given in 1.11 after some preparation in $1.9,1.10$. 
1.9. For $g \in \mathcal{G}$ we set $T_{g}=\mathcal{Z}_{Z_{\mathcal{G}}\left(g_{s}\right)}^{0}$, a torus contained in $Z_{\mathcal{G}}(g)$. For $g, g^{\prime} \in \mathcal{G}$ we write $g \sim g^{\prime}$ if $g^{\prime} g^{-1} \in T_{g}=T_{g^{\prime}}$. This is an equivalence relation on $\mathcal{G}$. Note that if $g \sim g^{\prime}$ then $x g x^{-1} \sim x g^{\prime} x^{-1}$ for any $x \in \mathcal{G}$. We show:

(a) Let $g, g^{\prime} \in \mathcal{G}$ be such that $g \sim g^{\prime}$. Then $Z_{\mathcal{G}}(g)=Z_{\mathcal{G}}\left(g^{\prime}\right)$ and $g_{u}=g_{u}^{\prime}$.

The weaker statement that $Z_{\mathcal{G}}(g)^{0}=Z_{\mathcal{G}}\left(g^{\prime}\right)^{0}$ is contained in the proof of [L5, I, 3.4]. Let $s=g_{s}, u=g_{u}, s^{\prime}=g_{s}^{\prime}, u^{\prime}=g_{u}$. We have $g^{\prime}=t g$ where $t \in T_{g}=T_{g^{\prime}}$. Thus $s^{\prime} u^{\prime}=t s u$. Since $t \in Z_{\mathcal{G}}(g)$ we have $t s=s t, t u=u t$. Since $s, t$ are commuting semisimple elements, $t s$ is semisimple and it commutes with $u$. By uniqueness of the Jordan decomposition of $g^{\prime}$ we have $s^{\prime}=t s, u^{\prime}=u$. Let $x \in$ $Z_{\mathcal{G}}(g)$. Then $x s=s x$. We have $t \in \mathcal{Z}_{Z_{\mathcal{G}}(s)}$. Moreover, $x \in Z_{\mathcal{G}}(s)$, hence $x t=t x$ so that $x s^{\prime}=x t s=t s x=s^{\prime} x$. Thus $x \in Z_{\mathcal{G}}\left(s^{\prime}\right)$. We also have $x \in Z_{\mathcal{G}}(u)=Z_{\mathcal{G}}\left(u^{\prime}\right)$. Thus, $x \in Z_{\mathcal{G}}\left(s^{\prime} u^{\prime}\right)$ that is, $x \in Z_{\mathcal{G}}\left(g^{\prime}\right)$. Thus, $Z_{\mathcal{G}}(g) \subset Z_{\mathcal{G}}\left(g^{\prime}\right)$. By symmetry we have also $Z_{\mathcal{G}}\left(g^{\prime}\right) \subset Z_{\mathcal{G}}(g)$ hence $Z_{\mathcal{G}}(g)=Z_{\mathcal{G}}\left(g^{\prime}\right)$. This proves (a).

1.10. In this subsection we assume that we are in the setup of Proposition 1.8. Let $\pi: Z_{\mathcal{G}}(u) \rightarrow H$ be the homomorphism such that $\pi(x)=x$ for $x \in H$ and $\pi(x)=1$ for $x \in \mathcal{V}$. We show:

(a) Let $(x, \sigma),\left(x^{\prime}, \sigma^{\prime}\right),(y, \tau) \in \tilde{\Sigma}$. Assume that for some $v \in \mathcal{V}$ we have $v x u v^{-1} \sim$ $x^{\prime} u$. Then $Z_{H}(x)=Z_{H}\left(x^{\prime}\right)$. Assume further that $\sigma=\sigma^{\prime}$ (which makes sense by the previous sentence). Then $((x, \sigma),(y, \tau))=\left(\left(x^{\prime}, \sigma^{\prime}\right),(y, \tau)\right)$.

From $v x u v^{-1} \sim x^{\prime} u$ and $1.9(\mathrm{a})$ we see that $Z_{\mathcal{G}}\left(v x u v^{-1}\right)=Z_{\mathcal{G}}\left(x^{\prime} u\right)$. Since $\left(v x u v^{-1}\right)_{s}=v x v^{-1},\left(v x u v^{-1}\right)_{u}=u,\left(x^{\prime} u\right)_{s}=x^{\prime},\left(x^{\prime} u\right)_{u}=u$, we deduce that $Z_{\tilde{H}}\left(v x v^{-1}\right)=Z_{\tilde{H}}\left(x^{\prime}\right)$. Using the semidirect product decompositions $Z_{\mathcal{G}}(u)=$ $\left(v H v^{-1}\right) \mathcal{V}, Z_{\mathcal{G}}(u)=H \mathcal{V}$ we deduce

$$
Z_{v H v^{-1}}\left(v x v^{-1}\right) Z_{\mathcal{V}}\left(v x v^{-1}\right)=Z_{H}\left(x^{\prime}\right) Z_{\mathcal{V}}\left(x^{\prime}\right)
$$

that is

$$
v Z_{H}(x) v^{-1} Z_{\mathcal{V}}\left(v x v^{-1}\right)=Z_{H}\left(x^{\prime}\right) Z_{\mathcal{V}}\left(x^{\prime}\right) .
$$

Applying $\pi$, we deduce $Z_{H}(x)=Z_{H}\left(x^{\prime}\right)$. This proves the first assertion of (a). From this we see that for $z \in H$ we have $z^{-1} y z \in Z_{H}(x)$ if and only if $z^{-1} y z \in Z_{H}\left(x^{\prime}\right)$. In other words, we have $A_{x, y}=A_{x^{\prime}, y}$. It follows that for any connected component $h$ of $H$ we have $A_{x, y}^{h}=A_{x^{\prime}, y}^{h}$. Let $z \in A_{x, y}=A_{x^{\prime}, y}$. Clearly, $\operatorname{tr}\left(z^{-1} y z, \sigma\right)=\operatorname{tr}\left(z^{-1} y z, \sigma^{\prime}\right)$ (recall that $\left.\sigma=\sigma^{\prime}\right)$. We claim that $\operatorname{tr}\left(z x z^{-1}, \tau\right)=$ $\operatorname{tr}\left(z x^{\prime} z^{-1}, \tau\right)$. We set $e:=x^{\prime} x^{-1} \in H$. From $v x u v^{-1} \sim x^{\prime} u$ we have

$$
\tilde{e}:=x^{\prime} u v u^{-1} x^{-1} v^{-1} \in T_{x^{\prime} u} .
$$

Hence

$$
z \tilde{e} z^{-1}=z e\left(x v x^{-1}\right) v^{-1} z^{-1} \in z T_{x^{\prime} u} z^{-1}=T^{\prime}
$$

where

$$
T^{\prime}:=z \mathcal{Z}_{Z_{\mathcal{G}}\left(x^{\prime}\right)}^{0} z^{-1}=\mathcal{Z}_{Z_{\mathcal{G}}\left(z x^{\prime} z^{-1}\right)}^{0}
$$


Now $y u \in Z_{\mathcal{G}}\left(z x^{\prime} z^{-1}\right)$ hence $T^{\prime} \subset Z_{\mathcal{G}}(y u)$ that is, $T^{\prime} \subset Z_{\tilde{H}}(y)=Z_{H}(y) Z_{\mathcal{V}}(y)$. It follows that $\pi\left(T^{\prime}\right)$ is a torus contained in $Z_{H}(y)$. Hence $\pi\left(T^{\prime}\right) \subset Z_{H}^{0}(y)$. Applying $\pi$ to $z e\left(x v x^{-1}\right) v^{-1} z^{-1} \in T^{\prime}$ we obtain $z e z^{-1} \in \pi\left(T^{\prime}\right)$ hence $z e z^{-1} \in Z_{H}^{0}(y)$. We have $z x^{\prime} z^{-1}=\left(z e z^{-1}\right)\left(z x z^{-1}\right)$ where $z e z^{-1} \in Z_{H}^{0}(y)$ acts trivially on $\tau$. Hence $\operatorname{tr}\left(z x z^{-1}, \tau\right)=\operatorname{tr}\left(z x^{\prime} z^{-1}, \tau\right)$ as claimed. It follows that (a) holds.

Next we show:

(b) Let $\chi \in \hat{\Lambda}$. Let $(x, \sigma) \in \tilde{\Sigma} \chi, x^{\prime} \in \Sigma$. Assume that for some $g \in \mathcal{G}$ we have gxug ${ }^{-1} \sim x^{\prime} u$. Then there exists $\sigma^{\prime} \in I_{x^{\prime}}^{\chi}$ such that for any $(y, \tau) \in \tilde{\Sigma}$ we have $((x, \sigma),(y, \tau))=\left(\left(x^{\prime}, \sigma^{\prime}\right),(y, \tau)\right)$.

From 1.9(a) we have $\left(g x u g^{-1}\right)_{u}=u$ hence $g u g^{-1}=u$ that is $g \in Z_{\mathcal{G}}(u)$. We write $g=v g_{1}$ with $g_{1} \in H, v \in \mathcal{V}$. We have $g_{1} x u g_{1}^{-1}=x^{\prime \prime} u$ where $x^{\prime \prime} \in \Sigma$ and $v x^{\prime \prime} u v^{-1} \sim x^{\prime} u$. From 1.3 we have $\left.\left(\left(x^{\prime \prime}, \sigma^{\prime}\right),(y, \tau)\right)=(x, \sigma),(y, \tau)\right)$ where $\sigma^{\prime}=$ ${ }^{g_{1}} \sigma \in I_{x^{\prime \prime}}^{\chi}$. From $v x^{\prime \prime} u v^{-1} \sim x^{\prime} u$ and (a) we have $I_{x^{\prime \prime}}^{\chi}=I_{x^{\prime}}^{\chi}$ and $\left(\left(x^{\prime \prime}, \sigma^{\prime}\right),(y, \tau)\right)=$ $\left(\left(x^{\prime}, \sigma^{\prime}\right),(y, \tau)\right)$. Thus $((x, \sigma),(y, \tau))=\left(\left(x^{\prime}, \sigma^{\prime}\right),(y, \tau)\right)$. This proves $(\mathrm{b})$.

1.11. We now prove Proposition 1.8. For $g, g^{\prime} \in \mathcal{G}$ we write $g \asymp g^{\prime}$ if $\gamma g \gamma^{-1} \sim g^{\prime}$ for some $\gamma \in \mathcal{G}$. This is an equivalence relation on $\mathcal{G}$; the equivalence classes are called the strata of $\mathcal{G}$. According to [L5, I, 3.7], $\mathcal{G}$ has only finitely many strata. Let $S_{1}, S_{2}, \ldots, S_{n}$ be the strata of $\mathcal{G}$ which have nonempty intersection with $u \Sigma$. For each $i \in[1, n]$ we choose $x_{i} \in \Sigma$ such that $u x_{i} \in S_{i}$. Now let $(x, \sigma) \in \tilde{\Sigma}^{\chi}$. Let $S$ be the stratum of $\mathcal{G}$ that contains $x u$. We have $S=S_{i}$ for some $i \in[1, n]$. Hence $g x u g^{-1} \sim x_{i} u$ for some $g \in \mathcal{G}$. By 1.10(b) there exists $\sigma^{\prime} \in I_{x_{i}}^{\chi}$ such that $(x, \sigma)=\left(x_{i}, \sigma^{\prime}\right)$ in $\overline{\mathbf{V}}_{\chi^{\prime}}^{\chi}$. We see that $\mathfrak{A}_{\chi^{\prime}}^{\chi}$ is the image under $V^{\chi} \rightarrow \overline{\mathbf{V}}_{\chi^{\prime}}^{\chi}$ of the finite set consisting of $\left(x_{i}, \sigma^{\prime}\right)$ with $i \in[1, n], \sigma^{\prime} \in I_{x_{i}}^{\chi}$. The proposition is proved.

1.12. In this subsection we assume that $H=P G L_{2}(\mathbf{C}), \Lambda=\{1\}$ and that $\kappa$ is the standard weight function. (This example is as in 1.7(a) for a suitable $u$ with $\mathcal{G}$ of type $F_{4}$, see A.11.)

Let $\lambda \mapsto g_{\lambda}$ be an isomorphism of $\mathbf{C}^{*}$ onto a maximal torus $T$ of $H$ whose normalizer in $H$ is denoted by $N(T)$. Then any semisimple element of $H$ is conjugate to an element of the form $g_{\lambda}$, for some $\lambda \in \mathbf{C}^{*}$. We have $Z_{H}(1)=H$, $Z_{H}\left(g_{-1}\right)=N(T), Z_{H}\left(g_{\lambda}\right)=T$ if $\lambda \in \mathbf{C}^{*}-\{1,-1\}$. Hence $|\bar{Z}(1)|=1,\left|\bar{Z}\left(g_{-1}\right)\right|=$ $2,\left|\bar{Z}\left(g_{\lambda}\right)\right|=1$ if $\lambda \in \mathbf{C}^{*}-\{1,-1\}$. Let $r \in N(T)-T$. We can find $\xi \in H$ such that $\xi g_{-1} \xi^{-1}=r$. We have $A_{1, g_{\lambda}}=H$ if $\lambda \in \mathbf{C}^{*}, A_{g_{-1}, g_{-1}}=T \cup r T \cup T \xi T \cup T \xi r T$, $A_{g_{-1}, g_{\lambda}}=T \cup r T$ if $\lambda \in \mathbf{C}^{*}-\{1,-1\}, A_{g_{\lambda}, g_{\lambda^{\prime}}}=T \cup r T$ if ${ }^{\prime} \lambda, \lambda^{\prime} \in \mathbf{C}^{*}-\{1,-1\}$. Hence we can take

$$
\begin{gathered}
{ }^{0} A_{1, g_{\lambda}}^{H}=\{1\},{ }^{0} A_{g_{-1}, g_{-1}}^{H}=\{1, r, \xi, \xi r\}, \\
{ }^{0} A_{g_{-1}, g_{\lambda}}^{H}=\{1, r\} \text { if } \lambda \in \mathbf{C}^{*}-\{1,-1\}, \\
{ }^{0} A_{g_{\lambda}, g_{\lambda^{\prime}}}^{H}=\{1, r\} \text { if } \lambda \in \mathbf{C}^{*}-\{1,-1\} .
\end{gathered}
$$

In our case any pair of commuting semisimple elements is adapted except for a pair $H$-conjugate to the pair $g_{-1}, r$. We have:

$$
\kappa\left(1, g_{\lambda}, H\right)=1, \kappa\left(g_{-1}, g_{-1}\right)=1 / 2,
$$




$$
\begin{gathered}
\kappa\left(g_{-1}, g_{\lambda}\right)=1 / 2 \text { if } \lambda \in \mathbf{C}^{*}-\{1,-1\}, \\
\kappa\left(g_{\lambda}, g_{\lambda^{\prime}}\right)=1 / 2 \text { if } \lambda \in \mathbf{C}^{*}-\{1,-1\} .
\end{gathered}
$$

Let $\epsilon$ be the non-trivial 1-dimensional representation of $N(T)$ which is trivial on $T$. From the previous results we can write the $4 \times 4$ matrix of inner products $((x, \sigma),(y, \tau))$ with $(x, \sigma)$ running through $(1,1),\left(g_{-1}, 1\right),\left(g_{-1}, \epsilon\right),\left(g_{\lambda}, 1\right)$ (they index the rows), $(y, \tau)$ running through $(1,1),\left(g_{-1}, 1\right),\left(g_{-1}, \epsilon\right),\left(g_{\lambda^{\prime}}, 1\right)$ (they index the columns) and with $\lambda, \lambda^{\prime} \in \mathbf{C}^{*}-\{1,-1\}$ :

$$
\left(\begin{array}{cccc}
1 & \frac{1}{2} & \frac{1}{2} & 1 \\
\frac{1}{2} & \frac{1}{2} & 0 & \frac{1}{2} \\
\frac{1}{2} & 0 & \frac{1}{2} & \frac{1}{2} \\
1 & \frac{1}{2} & \frac{1}{2} & 1
\end{array}\right)
$$

We see that for any $\lambda \in \mathbf{C}^{*}-\{1,-1\}$ we have $\left(g_{\lambda}, 1\right)=(1,1)$ in $\overline{\mathbf{V}}_{1}^{1}$. Also, $(1,1)=\left(g_{-1}, 1\right)+\left(g_{-1}, \epsilon\right)$ in $\overline{\mathbf{V}}_{1}^{1}$. Hence $\left(g_{-1}, 1\right),\left(g_{-1}, \epsilon\right)$ generate $\overline{\mathbf{V}}_{1}^{1}$ and the matrix of their inner products is

$$
\left(\begin{array}{cc}
\frac{1}{2} & 0 \\
0 & \frac{1}{2}
\end{array}\right)
$$

which is nonsingular, so that $\mathfrak{A}_{1}^{1}$ is equal to $\left\{\left(g_{-1}, 1\right),\left(g_{-1}, \epsilon\right)\right\}$ and is a basis of $\overline{\mathbf{V}}_{1}^{1}$.

\section{A CONJECTURE}

2.1. Let $\mathbf{k}$ be an algebraic closure of a finite field $F_{q}$. Let $K=\mathbf{k}((t))$ where $t$ is an indeterminate. Let $K_{0}=F_{q}((t))$, a subfield of $K$. Let $G$ be a simple adjoint algebraic group defined and split over $F_{q}$. Let $\mathcal{G}$ be a connected, simply connected, almost simple reductive group over $\mathbf{C}$ of type dual to that of $G$. Let $P_{\emptyset}$ be an Iwahori subgroup of $\mathbf{G}:=G(K)$ such that $P_{\emptyset} \cap G\left(K_{0}\right)$ is an Iwahori subgroup of $G\left(K_{0}\right)$. Let $\mathbf{W}$ be an indexing set for the set of $\left(P_{\emptyset}, P_{\emptyset}\right)$-double cosets in $\mathbf{G}$. We regard $\mathbf{W}$ as a group as in [L4]. As in loc.cit., $\mathbf{W}$ is the semidirect product of a (normal) subgroup $\mathbf{W}^{\prime}$ (an affine Weyl group) and a finite abelian subgroup $\Omega$. For any $\xi \in \Omega$ let $^{\xi} \mathbf{G}$ be the union of the $\left(P_{\emptyset}, P_{\emptyset}\right)$ double cosets in $\mathbf{G}$ indexed by elements in $\mathbf{W}^{\prime} \xi$. We have $\mathbf{G}=\sqcup_{\xi \in \Omega} \xi \mathbf{G}$. Let $\mathbf{G}_{r s c}$ be the set of regular semisimple compact elements of $\mathbf{G}$, see [L6, 3.1]. We have $\mathbf{G}_{r s c}=\sqcup_{\xi \in \Omega}{ }^{\xi} \mathbf{G}_{r s c}$ where ${ }^{\xi} \mathbf{G}_{r s c}={ }^{\xi} \mathbf{G} \cap \mathbf{G}_{r s c}$. We set ${ }^{\xi} G\left(K_{0}\right)_{r s c}={ }^{\xi} \mathbf{G}_{r s c} \cap G\left(K_{0}\right)$. Let $\mathcal{U}$ be the set of isomorphism classes of unipotent representations of $G\left(K_{0}\right)$, see [L4]. To each $\rho \in \mathcal{U}$ we attach a sign $\Delta(\rho) \in\{1,-1\}$ as follows: from the definition, to $\rho$ corresponds a unipotent cuspidal representation $\rho_{0}$ of a finite reductive group and $\Delta(\rho)$ is the invariant $\Delta\left(\rho_{0}\right)$ defined in $[\mathrm{L} 2,4.23,4.21]$. (We almost always have $\Delta(\rho)=1$.) Recall that [L4] gives a bijection $\mathfrak{Z}^{1} \leftrightarrow \mathcal{U}$ where for $\xi \in \Omega, \mathfrak{Z}^{\xi}$ is the set of all pairs $(g, \sigma)$ (up to $\mathcal{G}$-conjugation) where $g \in \mathcal{G}$ and $\sigma$ is an irreducible representation of 
$Z_{\mathcal{G}}(g) / Z_{\mathcal{G}}(g)^{0}$ on which $\mathcal{Z}_{\mathcal{G}}$ acts according to $\xi$ (we can identify $\Omega=\operatorname{Hom}\left(\mathcal{Z}_{\mathcal{G}}, \mathbf{C}^{*}\right)$ ). For $\zeta \in \mathfrak{Z}^{1}$ let $R_{\zeta}$ be the corresponding unipotent representation, let $\hat{R}_{\zeta}$ be the corresponding standard representation of $G\left(K_{0}\right)$ (which has $R_{\zeta}$ as a quotient) and let $\phi_{\zeta}^{\xi}$ be $\Delta\left(R_{\zeta}\right)$ times the restriction of the character of $\hat{R}_{\zeta}$ to ${ }^{\xi} G\left(K_{0}\right)_{r s c}$. For any $\zeta \in \mathfrak{Z}^{\xi}$ let $\mathbf{t}_{\zeta}:{ }^{\xi} G\left(K_{0}\right)_{r s c} \rightarrow \mathbf{C}$ be the unipotent almost character defined as in $[\mathrm{L} 6,3.9]$ (we assume that [L6, 3.8(f)] holds).

We now fix a unipotent element $u$ of $\mathcal{G}$ and a reductive subgroup $H$ of $Z_{\mathcal{G}}(u)$ as in 1.7. Then the definitions in $\S 1$ can be applied to this $H$ and to $\Lambda=\mathcal{Z}_{\mathcal{G}}$ (a subgroup of $H$ ). In particular $\Sigma, \tilde{\Sigma}, \mathbf{V}, \overline{\mathbf{V}}_{\chi^{\prime}}^{\chi}, \mathfrak{A}_{\chi^{\prime}}^{\chi}$ and the pairing 1.3(b) are defined. If $(x, \sigma) \in \tilde{\Sigma}^{1}$ then $\sigma$ can be viewed as an irreducible representation of $Z_{\mathcal{G}}(g) / Z_{\mathcal{G}}(g)^{0}$ on which $\mathcal{Z}_{\mathcal{G}}$ acts trivially hence the $\mathcal{G}$-conjugacy class of $(s u, \sigma)$ can be viewed as an element $\zeta=\zeta_{x, \sigma} \in \mathfrak{Z}^{1}$. We write $\hat{R}_{x, \sigma}, \phi_{x, \sigma}^{\xi}, \mathbf{t}_{x, \sigma}$ instead of $\hat{R}_{\zeta}, \phi_{\zeta}^{\xi}, \mathbf{t}_{\zeta}$. (Note that $\mathbf{t}_{\zeta}$ is defined up to multiplication by a root of 1.) Let $\mathfrak{A}_{\xi}^{1}$ be the set of (class) functions ${ }^{\xi} G\left(K_{0}\right)_{r s c} \rightarrow \mathbf{C}$ of the form $\phi_{x, \sigma}^{\xi}$ for some $(x, \sigma) \in \tilde{\Sigma}^{1}$. Let $\underline{\mathbf{V}}_{\xi}(u)$ be the vector space of (class) functions ${ }^{\xi} G\left(K_{0}\right)_{r s c} \rightarrow \mathbf{C}$ spanned by $\underline{\mathfrak{A}}_{\xi}^{1}$.

Conjecture 2.2. We assume that $\kappa$ is the standard weight function for $H$.

(a) For any $\xi \in \Omega$ there is a unique isomorphism of vector spaces $\Theta_{\xi}: \overline{\mathbf{V}}_{\xi}^{1} \stackrel{\sim}{\longrightarrow}$ $\underline{\mathbf{V}}_{\xi}(u)$ such that for any $(x, \sigma) \in \tilde{\Sigma}^{1}$ we have $\Theta_{\xi}\left((x, \sigma)_{\xi}\right)=\phi_{x, \sigma}^{\xi}$. In particular $\Theta_{\xi}$ defines a bijection $\mathfrak{A}_{\xi}^{1} \stackrel{\sim}{\longrightarrow} \mathfrak{A}_{\xi}^{1}$.

(b) There is a unique subset $\mathfrak{B}_{1}^{1}$ of $\mathfrak{A}_{1}^{1}$ such that $\mathfrak{B}_{1}^{1}$ is a basis of $\overline{\mathbf{V}}_{1}^{1}$ and any element of $\mathfrak{A}_{1}^{1}$ is an $\mathbf{R}_{\geq 0}$-linear combination of elements in $\mathfrak{B}_{1}^{1}$.

(c) Let $(x, \sigma) \in \tilde{\Sigma}^{1}$. We set $(x, \sigma)_{1}^{*}=\sum_{b \in \mathfrak{B}_{1}^{1}}\left(b,(x, \sigma)_{1}\right) b \in \overline{\mathbf{V}}_{1}^{1}$. We have $\Theta_{1}\left((x, \sigma)_{1}^{*}\right)=\mathbf{t}_{x, \sigma}$ up to a nonzero scalar factor.

\section{AN EXAMPLE}

3.1. We preserve the setup of 2.1 and we assume that $G$ is of type $C_{n}$ with $n \geq 2$; then we can take $\mathcal{G}=\operatorname{Spin}_{2 n+1}(\mathbf{C})$. We take $u$ in 2.1 to be a subregular unipotent element in $\mathcal{G}$ so that $H$ in 2.1 is as in 1.5 (with $r^{2}=g_{-1}^{n}$ ). We take $\kappa$ to be the standard weight function for $H$.

The simple reflections of the affine Weyl group $\mathbf{W}^{\prime}$ in 2.1 are denoted by $s_{0}, s_{1}, \ldots, s_{n}$ where $s_{i} s_{i+1}$ has order 4 if $i=0$ or $i=n-1$ and order 3 if $0<i<n-1 ; s_{i}, s_{j}$ commute if $|i-j| \geq 2$. For any $i \in[0, n]$ let $\mathbf{W}^{\prime i}$ be the subgroup of $\mathbf{W}^{\prime}$ generated by $\left\{s_{j} ; j \in[0, n]-\{i\}\right\}$; this can be viewed as the Weyl group of the reductive quotient $G^{i}$ of a maximal parahoric subgroup of $\mathbf{G}$ containing $P_{\emptyset}$. Note that $G^{i}$ is defined over $F_{q}$.

Let $\tilde{\mathcal{H}}_{q}$ be the extended affine Hecke algebra (over $\mathbf{C}$ ) with parameter $q$ corresponding to $\mathbf{W}$ and let $\mathcal{H}_{q}$ be unextended affine Hecke algebra with parameter $q$ corresponding to $\mathbf{W}^{\prime}$ (a subalgebra of $\tilde{\mathcal{H}}_{q}$ ); note that $\tilde{\mathcal{H}}_{q}$ contains an element $\omega$ such that $\omega^{2}=1, \tilde{\mathcal{H}}_{q}=\mathcal{H}_{q} \oplus \mathcal{H}_{q} \omega$ and such that $x \mapsto \omega x \omega$ is the algebra automorphism of $\mathcal{H}_{q}$ induced by the automorphism of $\mathbf{W}^{\prime}$ given by $s_{i} \mapsto s_{n-i}$ for $i \in[0, n]$. 
For any $i \in[0, n]$ let $\mathcal{H}_{q}^{i}$ be the Hecke algebra with parameter $q$ corresponding to $\mathbf{W}^{\prime i}$, viewed naturally as a subalgebra of $\mathcal{H}_{q}$.

We consider the following $\mathbf{W}^{\prime}$-graphs (in the sense of $[\mathrm{KL}]$ but with the $\mu$ function allowed to take complex values):

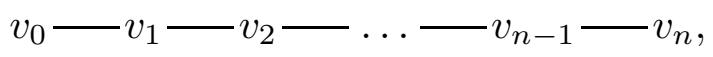

$$
v_{1}-v_{2}-\ldots-v_{n-1},
$$

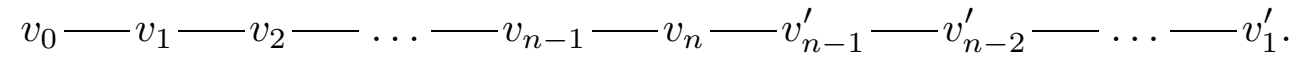

$\left(\operatorname{In}\left(d_{\lambda}\right), v_{1}^{\prime}\right.$ is also joined with $\left.v_{0}.\right)$

In each case the vertex $v_{j}$ or $v_{j}^{\prime}$ has associated set of simple reflections $\left\{s_{j}\right\}$. For (a) we have $\mu\left(v_{0}, v_{1}\right)=2, \mu\left(v_{n}, v_{n-1}\right)=2$, all other $\mu$ are equal to 1 . For (b) all $\mu$ are equal to 1 . For (c) there are no edges hence no $\mu$-function. For $\left(d_{\lambda}\right)$ with $\lambda \in \mathbf{C}^{*}$ we have $\mu\left(v_{0}, v_{1}\right)=\lambda, \mu\left(v_{1}, v_{0}\right)=\lambda^{-1}, \mu\left(v_{n}, v_{n-1}^{\prime}\right)=\lambda, \mu\left(v_{n-1}^{\prime}, v_{n}\right)=\lambda^{-1}$, all other $\mu$ are equal to 1 . For any $i \in[0, n]$, the $\mathbf{W}^{\prime}$-graphs (a),(b),(c) give rise to a $\mathbf{W}^{\prime i}$-graph with the same vertices (except the one marked with $s_{i}$ ), same $\mu$-function and same associated set of simple reflections (at any non-removed vertex). This $\mathbf{W}^{\prime i}$-graph is denoted by $\left(a^{i}\right),\left(b^{i}\right),\left(c^{i}\right)$ respectively. Let $n_{a}^{i}, n_{b}^{i}, n_{c}^{i}$ be the number of vertices marked with $s_{i}$ in (a),(b),(c) respectively (this number is 1 or 0 ).

For each of the $\mathbf{W}^{\prime}$-graphs $(\mathrm{a}),(\mathrm{b}),(\mathrm{c}),\left(d_{\lambda}\right)$ we consider the $\mathbf{C}$-vector space $E^{a}, E^{b}, E^{c}, E^{d_{\lambda}}$ spanned by the vertices of the graph, viewed as a $\mathcal{H}_{q}$-module in the standard way defined by the $\mathbf{W}^{\prime}$-graph structure. This $\mathcal{H}_{q}$-module extends to a $\tilde{\mathcal{H}}_{q}$-module in which $\omega$ acts by permuting the bases elements according to: $v_{i} \leftrightarrow v_{n-i}$ (for (a),(b),(c)) and (in case $\left.\left(d_{\lambda}\right)\right), v_{i} \leftrightarrow v_{n-i}^{\prime}$ if $i \in[1, n-1]$, $v_{0} \leftrightarrow v_{n}$. Let $\hat{E}^{a}, \hat{E}^{b}, \hat{E}^{c}, \hat{E}^{d_{\lambda}}$ be the admissible representations of $G\left(K_{0}\right)$ generated by their subspace of $P_{\emptyset} \cap G\left(K_{0}\right)$-invariant vectors such that the natural $\tilde{\mathcal{H}}_{q}$-module structure on this subspace is $E^{a}, E^{b}, E^{c}, E^{d_{\lambda}}$ respectively. Now the $\tilde{\mathcal{H}}_{q}$-modules $E^{a}, E^{b}, E^{c}, E^{d_{\lambda}}$ can be specialized to $q=1$ (using the $\mathbf{W}^{\prime}$-graph structure) and yield $\mathbf{W}$-modules $E_{1}^{a}, E_{1}^{b}, E_{1}^{c}, E_{1}^{d_{\lambda}}$ respectively.

If $i \in[0, n]$ for each of the $\mathbf{W}^{\prime i}$-graphs $\left(a^{i}\right),\left(b^{i}\right),\left(c^{i}\right)$ we consider the $\mathbf{C}$-vector space $E^{a^{i}}, E^{b^{i}}, E^{c^{i}}$ spanned by the vertices of the graph, viewed as a $\mathcal{H}_{q}^{i}$-module in the standard way defined by the $\mathbf{W}^{\prime i}$-graph structure. The restriction of the $\mathcal{H}_{q^{-}}$ module $E^{a}, E^{b}, E^{c}$ to $\mathcal{H}_{q}^{i}$-module is isomorphic to $E^{a^{i}} \oplus \mathbf{C}^{n_{a}^{i}}, E^{b^{i}} \oplus \mathbf{C}^{n_{b}^{i}}, E^{c^{i}} \oplus \mathbf{C}^{n_{c}^{i}}$ respectively. Here $\mathbf{C}^{m}$ is 0 if $m=0$ while if $m=1$ it is the $\mathcal{H}_{q}^{i}$-module defined by the $\mathbf{W}^{\prime i}$ graph with a single vertex without any associated simple reflection. 
Let $\hat{E}^{a^{i}}, \hat{E}^{b^{i}}, \hat{E}^{c^{i}}$ be the representations of $G^{i}\left(F_{q}\right)$ generated by their subspace of vectors invariant under a Borel subgroup such that the natural $\mathcal{H}_{q}^{i}$-module structure on this subspace is $E^{a^{i}}, E^{b^{i}}, E^{c^{i}}$ respectively. Now the $\mathcal{H}_{q}^{i}$-modules $E^{a^{i}}, E^{b^{i}}, E^{c^{i}}$ can be specialized to $q=1$ (using the $\mathbf{W}^{\prime i}$-graph structure) and yield $\mathbf{W}^{\prime i}$-modules $E_{1}^{a^{i}}, E_{1}^{b^{i}}, E_{1}^{c^{i}}$ respectively.

With notation in 2.1 and $1.5, \hat{E}^{a}, \hat{E}^{b}, \hat{E}^{c}$ can be identified with the standard representations $\hat{R}_{1,1}, \hat{R}_{1, \epsilon}, \hat{R}_{r, 1}$ of $G\left(K_{0}\right)$. On the other hand the standard representation $\hat{R}_{1, g_{\nu}}$ (where $\nu \in \mathbf{C}^{*}-\{1,-1\}$ ) is among the representations $\hat{E}^{d_{\lambda}}$. Let $\phi_{1,1}^{*}, \phi_{1, \epsilon}^{*}, \phi_{r, 1}^{*}, \phi_{1, g_{\nu}}^{*}$ be the restriction of the character of $\hat{R}_{1,1}, \hat{R}_{1, \epsilon}, \hat{R}_{r, 1}, \hat{R}_{1, g_{\nu}}$ to the set $G\left(K_{0}\right)_{\text {vrc }}$ of very regular compact elements (see [KmL]) in $G\left(K_{0}\right)$. According to $[\mathrm{KmL}], G\left(K_{0}\right)_{v r c}$ has a canonical partition $\sqcup_{\theta} G\left(K_{0}\right)_{v r c}^{\theta}$ into pieces invariant under conjugation by $G\left(K_{0}\right)$, where $\theta$ runs over the set of $\mathbf{W}$-conjugacy classes of elements of finite order in $\mathbf{W}^{\prime}$. Any finite dimensional $\mathbf{W}$-module $\rho$ gives rise to a class function $e_{\rho}: G\left(K_{0}\right)_{v r c} \rightarrow \mathbf{C}$ where $\left.e_{\rho}\right|_{G\left(K_{0}\right)_{v r c}^{\theta}}$ is the constant equal to the value of $\rho$ at any element of $\theta$. In particular the class functions $e_{E_{1}^{a}}, e_{E_{1}^{b}}, e_{E_{1}^{c}}$ on $G\left(K_{0}\right)_{\text {vrc }}$ are well defined; we denote them by $e_{1,1}, e_{1, \epsilon}, e_{r, 1}$. We set $e_{r, \epsilon}=0$ (a function on $\left.G\left(K_{0}\right)_{v r c}\right)$.

We have:

$$
\phi_{1, g_{\nu}}^{*}=\phi_{1,1}^{*}+\phi_{1, \epsilon}^{*} .
$$

To prove (e) it is enough to show that $\hat{E}^{d_{\lambda}}$ and $\hat{E}^{a} \oplus \hat{E}^{b}$ have the same character on $G\left(K_{0}\right)_{v r c}$ for any $\lambda \in \mathbf{C}^{*}$. By the results of $[\mathrm{KmL}]$, the character of $\hat{E}^{d_{\lambda}}$ on $G\left(K_{0}\right)_{v r c}$ depends only on the restrictions of $E^{d_{\lambda}}$ to the various $\mathcal{H}_{q}^{i}$ and in particular is independent of $\lambda$. Hence we can assume that $\lambda=1$. Thus it is enough to show that $E^{d_{1}} \cong E^{a} \oplus E^{b}$ as a $\mathcal{H}_{q}$-module. The $\mathbf{W}^{\prime}$-graph $\left(d_{1}\right)$ admits an involution $v_{i} \leftrightarrow v_{i}^{\prime}(i \in[1, n-1]), v_{0} \mapsto v_{0}, v_{n} \mapsto v_{n}$. Its eigenspaces give the required direct sum decomposition of the $\mathcal{H}_{q}$-module $E^{d_{1}}$.

We have the following equalities.

$$
\begin{aligned}
& \phi_{1,1}^{*}=1 / 2\left(e_{1,1}+e_{1, \epsilon}+e_{r, 1}+e_{r, \epsilon}\right), \\
& \phi_{1, \epsilon}^{*}=1 / 2\left(e_{1,1}+e_{1, \epsilon}-e_{r, 1}-e_{r, \epsilon}\right), \\
& \phi_{r, 1}^{*}=1 / 2\left(e_{1,1}-e_{1, \epsilon}+e_{r, 1}-e_{r, \epsilon}\right) .
\end{aligned}
$$

To prove (f) it is enough to show that for any $i \in[0, n]$ and any conjugacy class $\theta_{0}$ of $\mathbf{W}^{\prime i}$, the two sides of (f) take the same value on $G\left(K_{0}\right)_{v r c}^{\theta}$ where $\theta$ is the $\mathbf{W}$-conjugacy class in $\mathbf{W}^{\prime}$ that contains $\theta_{0}$. By $[\mathrm{KmL}],\left.\phi_{1,1}^{*}\right|_{G\left(K_{0}\right)_{v r c}^{\theta}}$ is the constant equal to $n_{a}^{i}$ plus the value of the character of $\hat{E}^{a^{i}}$ at a regular semisimple element 
of $G^{i}\left(F_{q}\right)$ of type $\theta_{0}$. By the results of [L2], this is equal to $n_{a}^{i}$ plus the character of $1 / 2\left(E_{1}^{a^{i}}+E_{1}^{b^{i}}+E_{1}^{c^{i}}\right)$ at $\theta_{0}$ hence it is equal to $n_{a}^{i}$ plus the character of $1 / 2\left(e_{1,1}-n_{a}^{i}+\right.$ $\left.e_{1, \epsilon}-n_{b}^{i}+e_{r, 1}-n_{c}^{i}+e_{r, \epsilon}\right)$ at $\theta$. It remains to note that $n_{a}^{i}=1 / 2\left(n_{a}^{i}+n_{b}^{i}+n_{c}^{i}\right)$. The proof of $(\mathrm{g})$ and $(\mathrm{h})$ is entirely similar; it is based on [KmL], [L2] and the equalities $n_{b}^{i}=1 / 2\left(n_{a}^{i}+n_{b}^{i}-n_{c}^{i}\right), n_{c}^{i}=1 / 2\left(n_{a}^{i}-n_{b}^{i}+n_{c}^{i}\right)$.

Now $\hat{R}_{r, \epsilon}$ is an irreducible representation of $G\left(K_{0}\right)$ which is not Iwahori-spherical. Let $\phi_{r, \epsilon}^{*}$ be the restriction of its character to $G\left(K_{0}\right)_{v r c}$. Using again [KmL], [L2], we see that

$$
\phi_{r, \epsilon}^{*}=1 / 2\left(e_{1,1}-e_{1, \epsilon}-e_{r, 1}+e_{r, \epsilon}\right) .
$$

From (f)-(i) we deduce:

$$
\begin{aligned}
& 1 / 2\left(\phi_{1,1}^{*}+\phi_{1, \epsilon}^{*}+\phi_{r, 1}^{*}+\phi_{r, \epsilon}^{*}\right)=e_{1,1}, \\
& 1 / 2\left(\phi_{1,1}^{*}+\phi_{1, \epsilon}^{*}-\phi_{r, 1}^{*}-\phi_{r, \epsilon}^{*}\right)=e_{1, \epsilon}, \\
& 1 / 2\left(\phi_{1,1}^{*}-\phi_{1, \epsilon}^{*}+\phi_{r, 1}^{*}-\phi_{r, \epsilon}^{*}\right)=e_{r, 1}, \\
& 1 / 2\left(\phi_{1,1}^{*}-\phi_{1, \epsilon}^{*}-\phi_{r, 1}^{*}+\phi_{r, \epsilon}^{*}\right)=e_{r, \epsilon} .
\end{aligned}
$$

which, together with (e), confirm the Conjecture 2.2 in a very special case.

\section{APPENDIX}

A.0. Let $\mathcal{G}, u, \mathcal{V}, H=H(u)$ be as in 1.7. In this appendix we give some information on the structure of $H$.

A.1. Assume first that $\mathcal{G}=S L(V)$ where $V$ is a $\mathbf{C}$-vector space of dimension $N \geq 2$. We assume that $V=\oplus_{i \geq 1} V_{i}$ where $V_{i}=W_{i} \otimes E_{i}$ and $W_{i}, E_{i}$ are $\mathbf{C}$ vector spaces of dimension $i, m_{i}$ respectively. Let $I=\left\{i \geq 1 ; m_{i} \geq 1\right\}$. For $i \in I$ we have an imbedding $\tau_{i}: G L\left(E_{i}\right) \rightarrow G L\left(V_{i}\right), g \mapsto 1_{W_{i}} \otimes g$. Let $\Phi$ : $\prod_{i \in I} G L\left(E_{i}\right) \rightarrow G L(V)$ be the homomorphism $\left(g_{i}\right) \mapsto \oplus_{i \in I} \tau_{i}\left(g_{i}\right)$ (an imbedding). Let $H=S L(V) \cap$ image of $\Phi$. Then $H$ is of the form $H(u)$ for some $u \in \mathcal{G}$ (as in 1.7) and all $H(u)$ as in 1.7 are obtained up to conjugacy. Note that $\Phi^{-1}(H)$ is the set of all $\left(x_{i}\right)_{i \in I}$ with $x_{i} \in G L\left(E_{i}\right), \prod_{i \in I} \operatorname{det}\left(x_{i}\right)^{i}=1$ and $\Phi^{-1}\left(\left(H^{0}\right)_{d e r}\right)$ is $\prod_{i \in I} S L\left(E_{i}\right)$. Thus $\left(H^{0}\right)_{d e r}$ is simply connected.

A.2. For any $\mathbf{C}$-vector space $V$ with a fixed symmetric or symplectic nondegenerate bilinear form $():, V \times V \rightarrow \mathbf{C}$ we denote by $I s_{V}$ be the group of isometries of $($,$) .$

Until the end of A.8 we assume that $V$ is a $\mathbf{C}$-vector space of dimension $N \geq 1$ with a fixed symmetric nondegenerate bilinear form $():, V \times V \rightarrow \mathbf{C}$; we write $O_{V}$ instead of $I s_{V}$. Let $V_{*}=\{v \in V ;(v, v)=1\}$. For any $v \in V_{*}$ define $r_{v} \in O_{V}$ by $r_{v}(v)=-v, r_{v}\left(v^{\prime}\right)=v^{\prime}$ if $v^{\prime} \in V,\left(v, v^{\prime}\right)=0$ (a reflection). 
Let $C(V)$ be the Clifford algebra of $($,$) that is, the quotient of the tensor algebra$ of $V$ by the two-sided ideal generated by the elements $v \otimes v^{\prime}+v^{\prime} \otimes v-2\left(v, v^{\prime}\right)$ with $v, v^{\prime} \in V$. As a vector space we have $C(V)=C(V)^{+} \oplus C(V)^{-}$where $C(V)^{+}$(resp. $C(V)^{-}$) is spanned by the products $v_{1} v_{2} \ldots v_{n}$ with $v_{i} \in V$ and $n$ even (resp. $n$ odd). Note that for $v \in V_{*}$ we have $v^{2}=1$ in $C(V)$. Let $\tilde{O}_{V}$ be the subgroup of the group of invertible elements of $C(V)$ consisting of the elements

$$
v_{1} v_{2} \ldots v_{n} \text { with } v_{i} \in V_{*}, n \geq 0 \text {. }
$$

Let ${ }^{0} \tilde{O}_{V}=\tilde{O}(V) \cap C(V)^{+},{ }^{1} \tilde{O}_{V}=\tilde{O}_{V} \cap C(V)^{-}=\tilde{O}_{V}-{ }^{0} \tilde{O}_{V}$. Then ${ }^{0} \tilde{O}_{V}$ is a subgroup of index 2 of $\tilde{O}_{V}$. We set $c=-1 \in C(V)$. If $v \in V,(v, v)=-1$, we have $v^{2}=-1$ in $C(V)$. Hence $c \in{ }^{0} \tilde{O}_{V}$. If $\xi \in \tilde{O}_{V}, v \in V$, we have $\xi v \xi^{-1} \in V$. (Indeed, for $\xi=v_{1} v_{2} \ldots v_{n}$ as in (a), we have $\xi v \xi^{-1}=(-1)^{n} r_{v_{1}} r_{v_{2}} \ldots r_{v_{n}}$.) Hence $\xi \mapsto\left[v \mapsto \xi v \xi^{-1}\right]$ is a homomorphism $\beta: \tilde{O}_{V} \rightarrow O_{V}$ with image $O_{V}$, if $N$ is even, and $O_{V}^{0}$ if $N$ is odd. The kernel of $\beta:{ }^{0} \tilde{O}_{V} \rightarrow O_{V}^{0}$ is $\{1, c\}$. If $N \geq 2$ we have ${ }^{0} \tilde{O}_{V}=\tilde{O}_{V}^{0}$. If $N=1,{ }^{0} \tilde{O}_{V}$ is of order 2 .

A.3. In the setup of A.2 we assume $\mathcal{G}={ }^{0} \tilde{O}_{V}$. We also assume (until the end of A.8) that $V=\oplus_{i \geq 1} V_{i}$ where $V_{i}=W_{i} \otimes E_{i}$ and $W_{i}, E_{i}$ are C-vector spaces of dimension $i, m_{i}$ respectively with given nondegenerate bilinear forms $($,$) (both$ symmetric if $i$ is odd, both symplectic if $i$ is even) such that $\left(w \otimes e, w^{\prime} \otimes e^{\prime}\right)=$ $\left(w, w^{\prime}\right)\left(e, e^{\prime}\right)$ for $w, w^{\prime} \in W_{i}, e, e^{\prime} \in E_{i}$ and $\left(V_{i}, V_{j}\right)=0$ for $i \neq j$.

Let $I=\left\{i \geq 1 ; m_{i} \geq 1\right\}, I_{\text {odd }}=I \cap(2 \mathbf{Z}+1), I_{\text {even }}=I \cap(2 \mathbf{Z})$. For any $t \geq 2$ let $I_{\text {odd }}^{\geq t}=\left\{i \in I_{\text {odd }} ; m_{i} \geq t\right\}$. For any $i \in I$ let $\beta_{i}: \tilde{O}_{V_{i}} \rightarrow O_{V_{i}}$ be the homomorphism defined like $\beta$ with $V$ replaced by $V_{i}$. For any $i \in I_{\text {odd }}$ let $w_{1}^{i}, w_{2}^{i}, \ldots, w_{i}^{i}$ be an orthonormal basis of $W_{i}$ with respect to $($,$) .$

In this appendix, any product over $I$ or $I_{\text {odd }}$ is taken using the order of $I$ or $I_{\text {odd }}$ induced from the obvious order on $\mathbf{N}$. The imbedding $\tau_{i}: I s_{E_{i}} \rightarrow O_{V_{i}}$, $g \mapsto 1_{W_{i}} \otimes g$, restricts to an imbedding $I s_{E_{i}}^{0} \rightarrow O_{V_{i}}^{0}$. Let $\Phi: \prod_{i \in I} I s_{E_{i}} \rightarrow O_{V}$ be the homomorphism $\left(g_{i}\right) \mapsto \oplus_{i \in I} \tau_{i}\left(g_{i}\right)$ (an imbedding); it restricts to an imbedding $\Phi^{0}: \prod_{i \in I} I s_{E_{i}}^{0} \rightarrow O_{V}^{0}$. Let $\bar{R}=O_{V}^{0} \cap$ image of $\Phi$. We have image of $\Phi^{0}=\bar{R}^{0}$.

For $i \in I_{o d d}$ we choose $e_{i} \in E_{i *}$ and we define $y_{i} \in O_{V}$ by $y_{i}=1$ on $W_{i} \otimes \mathbf{C} e_{i}$ and $y_{i}=-1$ on the perpendicular to $W_{i} \otimes \mathbf{C} e_{i}$ in $V$. Thus $y_{i}=\tau_{i}\left(-r_{e_{i}}\right)$ on $V_{i}, y_{i}=-1$ on $V_{j}$ for $j \neq i$. (Here $r_{e_{i}}$ is a reflection in $E_{i}$.) We see that $y_{i} \in$ image of $\Phi$, $y_{i}^{2}=1$.

Let $g=\oplus_{j \in I} \tau_{j}\left(\mathfrak{t}_{j}\right) \in O_{V}$ where $\mathfrak{t}_{j} \in I s_{E_{j}}$. From the definitions we see that for any $i \in I_{\text {odd }}$ we have

(a) $y_{i} g y_{i}^{-1}=\oplus_{j \in I} \tau_{j}\left(\mathfrak{t}_{j}^{\prime}\right) \in O_{V}$ where $\mathfrak{t}_{j}^{\prime} \in I s_{E_{j}}$ is given by $\mathfrak{t}_{i}^{\prime}=r_{e_{i}} \mathfrak{t}_{i} r_{e_{i}}$ and $\mathfrak{t}_{j}^{\prime}=\mathfrak{t}_{j}$ if $j \neq i$. In particular if $g \in \bar{R}^{0}$ then $y_{i} g y_{i}^{-1} \in \bar{R}^{0}$.

Let $\bar{\Gamma}$ be the (finite abelian) subgroup of $O_{V}$ generated by $\left\{y_{i} ; i \in I_{\text {odd }}\right\}$. Let $\bar{\Gamma}^{+}$be the subgroup of $\bar{\Gamma}$ consisting of elements which are products of an even number of generators $y_{i}$. If $I_{\text {odd }}=\emptyset$ we have $\bar{\Gamma}=\bar{\Gamma}^{+}=\{1\}$. If $I_{\text {odd }} \neq \emptyset$ then $\bar{\Gamma}^{+}$is a subgroup of index 2 of $\bar{\Gamma}$. We have $\bar{R}=\bar{R}^{0} \bar{\Gamma}^{+}$and $\bar{R}^{0} \cap \bar{\Gamma}^{+}=\{1\}$. Let 
$H=\{g \in \mathcal{G} ; \beta(x) \in \bar{R}\}$. Then $H$ is of the form $H(u)$ for some $u \in \mathcal{G}$ (as in 1.7) and all $H(u)$ as in 1.7 are obtained up to conjugacy.

A.4. Our next objective is to describe the structure of $H$, see A.6(e), A.7.

For $i \in I$ we set $S_{i}={ }^{0} \tilde{O}_{E_{i}}$ if $i \in I_{\text {odd }}$ and $S_{i}=I s_{E_{i}}$ if $i \in I_{\text {even }}$. For $i \in I$ let $R_{i}^{\prime}=\left\{\xi \in{ }^{0} \tilde{O}_{V_{i}} ; \beta_{i}(\xi) \in \tau_{i}\left(I s_{E_{i}}^{0}\right)\right\}$. We set $R_{i}=R_{i}^{\prime}$ if $i \in I_{o d d}$ and $R_{i}=R_{i}^{\prime 0}$ if $i \in I_{\text {even }}$.

For $i \in I_{\text {odd }}$ let $c_{i}^{\prime}$ be -1 , viewed as an element of $S_{i}$. For $i \in I$ let $c_{i}$ be -1 , viewed as an element of ${ }^{0} \tilde{O}\left(V_{i}\right)$; note that, if $i \in I_{\text {odd }}$, we have $c_{i} \in R_{i}$.

If $i \in I_{\text {odd }}$ then $R_{i}$ contains

$$
x_{i ; e, f}=\left(w_{i}^{1} \otimes e\right) \ldots\left(w_{i}^{i} \otimes e\right)\left(w_{i}^{1} \otimes f\right) \ldots\left(w_{i}^{i} \otimes f\right)=-x_{i ; f, e}
$$

for any $e, f \in E_{i *}$. (Indeed, $x_{i ; e, f}$ projects to $\left(1_{W_{i}} \otimes r_{e}\right)\left(1_{W_{i}} \otimes r_{f}\right)$, where $r_{e}, r_{f}$ are reflections in $E_{i}$.) Since $r_{e} r_{f}$ generate $\tau_{i}\left(O_{E_{i}}^{0}\right)$, we see that the elements $x_{i ; e, f}$ generate $R_{i}$.

If $i \in I_{o d d}$ and $m_{i}=1$ we have $\tau_{i}\left(I s_{E_{i}}^{0}\right)=\{1\}$ and $R_{i}=\left\{1, c_{i}\right\}$; we see that there is a unique isomorphism $S_{i} \stackrel{\sim}{\longrightarrow} R_{i}$. Thus $\tau_{i}^{0}$ lifts uniquely to an isomorphism $\tilde{t}_{i}: S_{i} \stackrel{\sim}{\longrightarrow} R_{i}$ which carries $c_{i}^{\prime}$ to $c_{i}$.

If $i \in I_{\text {odd }}^{\geq 2}$, from an argument in [L3, 14.3], we see that $R_{i}$ is connected; being a double covering of $O_{E_{i}}^{0}$, there is a unique isomorphism ${ }^{0} \tilde{O}_{E_{i}} \stackrel{\sim}{\longrightarrow} R_{i}$ which induces the identity on $O_{E_{i}}^{0}$. Thus $\tau_{i}^{0}$ lifts uniquely to an isomorphism $\tilde{t}_{i}: S_{i} \stackrel{\sim}{\longrightarrow} R_{i}$ which carries $c_{i}^{\prime}$ to $c_{i}$.

If $i \in I_{\text {even }}$ then, since $R_{i}^{\prime}$ is a double covering (with kernel $\left\{1, c_{i}\right\}$ ) of $S_{i}$ which is simply connected, we see that $R_{i}^{\prime}=R_{i} \sqcup c_{i} R_{i}$ and that $\tau_{i}$ lifts uniquely to an isomorphism $\tilde{t}_{i}: S_{i} \stackrel{\sim}{\longrightarrow} R_{i}$.

We set $S=\prod_{i \in I} S_{i}$; for any $s \in S$ we write $s_{i}$ for the $i$-component of $s$. Putting together the isomorphisms $\tilde{t}_{i}$ we get

$$
S \stackrel{\sim}{\longrightarrow} \prod_{i \in I} R_{i} \subset \prod_{i \in I}^{0} \tilde{O}_{V_{i}},
$$

Composing with the homomorphism

$$
\prod_{i \in I}{ }^{0} \tilde{O}_{V_{i}} \rightarrow \mathcal{G}
$$

induced by $\prod_{i \in I} C\left(V_{i}\right) \rightarrow C(V)$ (multiplication in $C(V)$ ) we obtain the homomorphism $\Phi^{\prime}$ in the commutative diagram

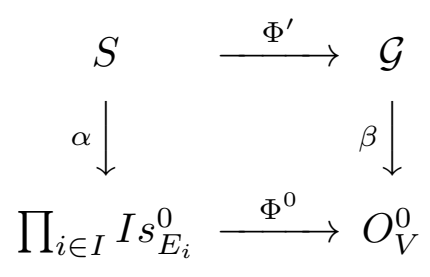


where $\alpha$ is the obvious homomorphism.

Let $R$ be the image of $\Phi^{\prime}$. Clearly, $R$ is a closed subgroup of $\mathcal{G}$. We show that $\beta(R)=\bar{R}^{0}$. Since $\alpha$ is surjective, this follows from (b) and the fact that the image of $\Phi^{0}$ is $\bar{R}^{0}$. Let

$$
A=\left\{s \in S ; s_{i}=1 \text { if } i \in I_{\text {even }}, s_{i}=c_{i}^{\prime n_{i}} \text { if } i \in I_{\text {odd }}, n_{i} \in \mathbf{Z} / 2, \sum_{i \in I_{\text {odd }}} n_{i}=0\right\}
$$

We show:

$$
\operatorname{Ker}\left(\Phi^{\prime}\right)=A
$$

From the definitions, if $s \in S$ is given by $s_{i}=1$ if $i \in I_{\text {even }}, s_{i}=c_{i}^{\prime n_{i}}$ if $i \in I_{\text {odd }}$ where $n_{i} \in \mathbf{Z} / 2$, then $\Phi^{\prime}(s)=c^{m}$ where $m=\sum_{i \in I_{\text {odd }}} n_{i}$. In particular, we have $A \subset \operatorname{Ker}\left(\Phi^{\prime}\right)$. Conversely, let $\left(\xi_{i}\right)_{i \in I} \in S$ be an element in $\operatorname{Ker}\left(\Phi^{\prime}\right)$. We set $\tilde{\xi}_{i}=\tilde{t}_{i}\left(\xi_{i}\right) \in R_{i}$. Viewing $\tilde{\xi}_{i}$ as an element of $C\left(V_{i}\right) \subset C(V)$, we have $\prod_{i \in I} \tilde{\xi}_{i}=1$ (product in $C(V)$ ). Let $I_{0}$ be the set of all $i \in I$ such that $\tilde{\xi}_{i}$ is a (nonzero) scalar. From the definition of $C(V)$ we see that, if $b_{i}^{j}\left(j \in\left[1,2^{\operatorname{dim} V_{i}}\right]\right.$ is a basis of $C\left(V_{i}\right)$, then the products $b^{f}:=\prod_{i \in I} b_{i}^{f(i)}$ (for various functions $f$ which to each $i \in I$ associate $\left.f(i) \in\left[1, \operatorname{dim} C\left(V_{i}\right)\right]\right)$ form a basis of $C(V)$. We can assume that $b_{i}^{1}=\tilde{\xi}_{i}$ for all $i \in I$ and $b_{i}^{2}=1$ for all $i \in I-I_{0}$. Let $f_{1}, f_{2}$ be such that $f_{1}(i)=1$ for all $i \in I, f_{2}(i)=2$ for $i \in I-I_{0}, f_{2}(i)=1$ for $i \in I_{0}$. We have $b^{f_{1}}=1, b^{f_{2}}=\lambda 1$ with $\lambda \in \mathbf{C}^{*}$. Thus $b^{f_{2}}=\lambda b^{f_{1}}$. Since $b^{f_{2}}, b^{f_{1}}$ are part of a basis of $C(V)$, we see that $f_{1}=f_{2}$ hence $I-I_{0}=\emptyset$. Thus $\tilde{\xi}_{i}$ is a nonzero scalar for any $i \in I$. It follows that the element $\tilde{\xi}_{i} \in{ }^{0} \tilde{O}_{V_{i}}$ satisfies $\beta_{i}\left(\tilde{\xi}_{i}\right)=1$ hence $\tilde{\xi}_{i}=c_{i}^{n_{i}}$ (hence $\xi_{i}=c_{i}^{\prime n_{i}}$ ) for some $n_{i} \in \mathbf{Z} / 2$. If in addition, $i \in I_{\text {even }}$, then $\tilde{\xi}_{i} \in R_{i}$ and $c_{i} \notin R_{i}$ implies that $n_{i}=0$. Now if $i \in I_{\text {odd }}$, then $c_{i}$ viewed as an element of $C(V)$ is equal to $c$. Hence from $\prod_{i \in I} \tilde{\xi}_{i}=1$ (in $\left.C(V)\right)$ it follows that $\sum_{i \in I_{\text {odd }}} n_{i}=0$. Thus $\operatorname{Ker}\left(\Phi^{\prime}\right) \subset A$. The opposite containment is obvious. This proves (c).

We show:

(d) If $I_{\text {odd }}^{\geq 2} \neq \emptyset$ then $R=R^{0}=H^{0}$ and $c \in H^{0}$.

If $i \in I$ and $m_{i} \geq 2$, then $R_{i}=R_{i}^{0}$. Hence

$$
\prod_{i \in I} R_{i}=\prod_{i \in I ; m_{i}=1} R_{i} \times \prod_{i \in I ; m_{i} \geq 2} R_{i}^{0}
$$

Thus $R$ is generated by $R^{0}$ and by $\Phi^{\prime}\left(c_{i}^{\prime}\right)$ for various $i \in I$ such that $m_{i}=1$. To show that $R=R^{0}$ it is enough to show that if $i \in I$ is such that $m_{i}=1$ (hence $i$ is odd) then $\Phi^{\prime}\left(c_{i}^{\prime}\right) \in R^{0}$. By assumption we can find $i^{\prime} \in I_{\text {odd }}^{\geq 2}$. From (c) we see that $\Phi^{\prime}\left(c_{i}^{\prime} c_{i^{\prime}}^{\prime}\right)=1$. Hence

$$
\Phi^{\prime}\left(c_{i}^{\prime}\right)=\Phi^{\prime}\left(c_{i^{\prime}}^{\prime}\right) \in \Phi^{\prime}\left(R_{i^{\prime}}\right)=\Phi^{\prime}\left(R_{i^{\prime}}^{0}\right) \subset R^{0}
$$


Thus we have $R=R^{0}$. Since $\beta(R)=\bar{R}^{0}, H=\beta^{-1}(\bar{R}) \cap \mathcal{G}$, we have $\operatorname{dim} R=$ $\operatorname{dim} H=\operatorname{dim} \bar{R}$ and $R \subset H$ so that $R^{0}=H^{0}$. From the proof of (c) we see that, if $s \in S$ is given by $s_{i}=c_{i}^{\prime}$ for $i=i^{\prime}$ (as above), $s_{i}=1$ for $i \in I-\left\{i^{\prime}\right\}$, then $\Phi^{\prime}(s)=c$. Thus, $c \in R$. Since $R=H^{0}$ we see that $c \in H^{0} ;(\mathrm{d})$ is proved.

We show:

(e) If $I_{\text {odd }}^{\geq 2} \neq \emptyset$ then $\beta$ induces an isomorphism $H / H^{0} \stackrel{\sim}{\longrightarrow} \bar{R} / \bar{R}^{0}=b a G^{+}$. Hence $H / H^{0}$ is an finite abelian group of order $2^{\left|I_{\text {odd }}-1\right|}$.

From A.3 we have $\bar{R} / \bar{R}^{0}=\bar{\Gamma}^{+}$. From the definitions, $\beta$ induces a surjective homomorphism $H \rightarrow \bar{R}$ with kernel $\{1, c\}$. It is then enough to note that $\{1, c\} \subset$ $H^{0}$ (see $\left.(\mathrm{d})\right)$.

A.5. Let $i \in I_{o d d}$. We define an automorphism $a_{i}: S \rightarrow S$ by $s \mapsto s^{\prime}$ where $s_{i}^{\prime}=e_{i} s_{i} e_{i}^{-1}$ (product in $\tilde{O}_{E_{i}}$ ), $s_{j}^{\prime}=s_{j}$ for $j \neq i$. Since $a_{i}$ induces the identity map on $\operatorname{Ker}\left(\Phi^{\prime}\right)$ (see A.4(c)), it follows that $a_{i}$ induces an automorphism $a_{i}^{\prime}: R \rightarrow R$ (recall that $R$ is the image of $\Phi^{\prime}$ ). We set

$$
\tilde{y}_{i}=\left(w_{i}^{1} \otimes e_{i}\right) \ldots\left(w_{i}^{i} \otimes e_{i}\right) \in \tilde{O}_{V}
$$

We show:

(a) For any $\tilde{g} \in R$ we have $\tilde{y}_{i} \tilde{g} \tilde{y}_{i}^{-1}=a_{i}^{\prime}(\tilde{g}) \in R$.

Assume first that $m_{i}=1$. We have $a_{i}=1$ (in this case, $e_{i}$ is in the centre of $O_{E_{i}}$ ). Now $R_{i}$ is generated by $x_{i ; e_{i}, e_{i}}=\tilde{y}_{i}^{2}$ (see A.4) and by $c_{i}$ hence $\tilde{y}_{i}$ commutes with any element of $R_{i}$; it also commutes with any element of $R_{j}, j \neq i$ hence $\tilde{y}_{i} \tilde{g} \tilde{y}_{i}^{-1}=\tilde{g}$. Thus (a) is proved in this case. Next we assume that $m_{i} \geq 2$. We have $\beta\left(\tilde{y}_{i}\right)=y_{i}$ hence

$$
\beta\left(\tilde{y}_{i} \tilde{g} \tilde{y}_{i}^{-1}\right)=y_{i} \beta(\tilde{g}) y_{i}^{-1} \in y_{i} \bar{R}^{0} y_{i}^{-1}=\bar{R}^{0}
$$

(see $(4.3(\mathrm{a})))$. Thus, $\tilde{y}_{i} R \tilde{y}_{i}^{-1} \in \beta^{-1}\left(\bar{R}^{0}\right) \cap \mathcal{G} \subset H$. Since $R=H^{0}$, see A.4(d), we see that $\tilde{y}_{i} R \tilde{y}_{i}^{-1}=\tilde{y}_{i} H^{0} \tilde{y}_{i}^{-1}$ is a connected subgroup of $H$. It follows that $\tilde{y}_{i} R \tilde{y}_{i}^{-1}=H^{0}=R$. Thus $A d\left(\tilde{y}_{i}\right)$ is an automorphism of $R$. From the definition and A.3(a) we have $\beta\left(a_{i}^{\prime}(\tilde{g})\right)=y_{i} \beta(\tilde{g}) y_{i}^{-1}=\beta\left(\tilde{y}_{i} \tilde{g} \tilde{y}_{i}^{-1}\right)$. Thus $\tilde{y}_{i} \tilde{g} \tilde{y}_{i}^{-1}=a_{i}^{\prime}(\tilde{g}) f(\tilde{g})$ where $f: R \rightarrow \operatorname{ker} \beta$ is a morphism. Now $R$ is connected (see A.4(d)) and ker $\beta$ is finite hence $f$ is constant. Clearly, $f(1)=1$. It follows that $f(\tilde{g})=1$ for any $\tilde{g} \in R$, proving (a).

A.6. Let $\Delta$ be the group defined by the generators $z_{i}\left(i \in I_{\text {odd }}\right)$ and $c_{*}$ with relations: $c_{*}^{2}=1, c_{*} z_{i}=z_{i} c_{*}, z_{i}^{2}=c_{*}^{i(i-1) / 2}$ for $i \in I_{o d d}$ and $z_{i} z_{i^{\prime}}=c_{*} z_{i^{\prime}} z_{i}$ for $i \neq i^{\prime}$ in $I_{o d d}$. (If $I_{o d d}=\emptyset, \Delta$ is the group of order 2 with generator $c_{*}$.) From the definition we see that any element of $\Delta$ can be written in the form $c_{*}^{n} \prod_{i \in I_{\text {odd }}} z_{i}^{n_{i}}$ where $n_{i} \in \mathbf{Z} / 2, n \in \mathbf{Z} / 2$. It follows that $|\Delta| \leq 2^{\left|I_{\text {odd }}\right|+1}$. The assignment $c_{*} \mapsto c$, $z_{i} \mapsto \tilde{y}_{i}=\left(w_{i}^{1} \otimes e_{i}\right) \ldots\left(w_{i}^{i} \otimes e_{i}\right) \in \tilde{O}_{V}$ defines a homomorphism $\zeta: \Delta \rightarrow \tilde{O}_{V}$. For any $i \in I_{\text {odd }}$ we have $\beta\left(\tilde{y}_{i}\right)=y_{i}$. Thus $\beta(\zeta(\Delta))$ is the subgroup of $O_{V}$ generated by $y_{i}\left(i \in I_{\text {odd }}\right)$, an (abelian) group of order $2^{\left|I_{\text {odd }}\right|}$. Since $\zeta(\Delta)$ contains $c$ which is in 
the kernel of $\beta$, we deduce that $|\zeta(\Delta)| \geq 2^{\left|I_{\text {odd }}\right|+1}$ hence $|\Delta|=|\zeta(\Delta)|=2^{\left|I_{\text {odd }}\right|+1}$. In particular, $\zeta$ defines an isomorphism $\Delta \stackrel{\sim}{\rightarrow} \zeta(\Delta)$ and any element of $\Delta$ can be written uniquely in the form $c_{*}^{n} \prod_{i \in I_{\text {odd }}} z_{i}^{n_{i}}$ where $n_{i} \in \mathbf{Z} / 2, n \in \mathbf{Z} / 2$.

We now define a homomorphism $\Delta \rightarrow \operatorname{Aut}(S), \delta \mapsto\left[s \mapsto{ }^{\delta} s\right]$ by $c_{*} \mapsto 1$, $z_{i} \mapsto a_{i}$ where $a_{i}$ is as in A.5. We form the semidirect product $S \cdot \Delta$ in which, for $s, s^{\prime} \in S, \delta, \delta^{\prime} \in \Delta$, we have $(s \delta)\left(s^{\prime} \delta^{\prime}\right)=\left(s^{\delta} s^{\prime}\right)\left(\mathbf{d}^{\prime}\right)$. We extend $\zeta: \Delta \rightarrow \tilde{O}_{V}$ to a homomorphism $S \cdot \Delta \rightarrow \tilde{O}_{V}$ (denoted again by $\zeta$ ) by definining $\zeta$ on $S$ to be $\Phi^{\prime}$. To show that this is well defined we must verify for $i \in I_{o d d}, s \in S$ that $\Phi^{\prime}\left(a_{i}(s)\right)=\tilde{y}_{i} \Phi^{\prime}(s) \tilde{y}_{i}^{-1}$. This follows from A.5(a). We show:

(a) The kernel of $\zeta: S \cdot \Delta \rightarrow \tilde{O}_{V}$ is equal to $A^{\prime}=\{1\}$ if $I_{\text {odd }}=\emptyset$ and to

$$
\begin{aligned}
& A^{\prime}:=\left\{s c_{*}^{n} ; s \in S, n \in \mathbf{Z} / 2, s_{i}=c_{i}^{\prime n_{i}} \text { if } i \in I_{\text {odd }}, s_{i}=1 \text { if } i \in I_{\text {even }},\right. \\
& \left.n_{i} \in \mathbf{Z} / 2, n=\sum_{i \in I_{\text {odd }}} n_{i}\right\}
\end{aligned}
$$

if $I_{\text {odd }} \neq \emptyset$.

Let $s \in S, \delta \in \Delta$ be such that $s \delta \in \operatorname{Ker}(\zeta)$. We write $\delta=c_{*}^{n} \prod_{i \in I_{\text {odd }}} z_{i}^{u_{i}}$ where $u_{i} \in \mathbf{Z} / 2, n \in \mathbf{Z} / 2$. We set $\xi_{i}=\tilde{t}_{i}\left(s_{i}\right) \in R_{i}$.

Assume first that $I_{\text {odd }} \neq \emptyset$. We have $1=c^{n} \prod_{i \in I} \xi_{i} \prod_{i \in I_{\text {odd }}} \tilde{y}_{i}^{u_{i}}$ hence $\prod_{i \in I} f_{i}=$ power of $c$, where $f_{i}=\tilde{\xi}_{i} \tilde{y}_{i}^{m_{i}}$ for $i \in I_{\text {odd }}, f_{i}=\xi_{i}$ for $i \in I_{\text {even }}$. We have $f_{i} \in$ $C\left(V_{i}\right)^{u_{i}}$ if $i \in I_{\text {odd }}$ and $f_{i} \in C\left(V_{i}\right)^{0}$ if $i \in I_{\text {even }}$. Now the subspaces $\prod_{i \in I} C\left(V_{i}\right)^{r_{i}}$ of $C(V)$ (with $r_{i} \in\{0,1\}$ ) form a direct sum decomposition of $C(V)$. It follows that $u_{i}=0$ for any $i \in I_{\text {odd }}$. Thus we have $c^{n} \prod_{i \in I} \xi_{i}=1$. If $n=0$ then from the previous equality we deduce as in the proof of A.5(c) that $s_{i}=c_{i}^{\prime n_{i}}$ if $i \in I_{\text {odd }}$, $s_{i}=1$ if $i \in I_{\text {even }}$ where $n_{i} \in \mathbf{Z} / 2, \sum_{i \in I_{\text {odd }}} n_{i}=0$. If $n=1$ we pick $i_{0} \in I_{\text {odd }}$ and we define $s^{\prime} \in S$ by $s_{i_{0}}^{\prime}=c_{i_{0}}^{\prime} s_{i_{0}}, s_{i}^{\prime}=s_{i}$ if $i \in I-\left\{i_{0}\right\}$. Then $\zeta\left(s^{\prime}\right)=1$ from which we deduce as above that $s_{i}^{\prime}=c_{i}^{\prime n_{i}}$ if $i \in I_{\text {odd }}$ with $n_{i} \in \mathbf{Z} / 2, \sum_{i \in I_{\text {odd }}} n_{i}=0$. Hence $s_{i}^{\prime}=c_{i}^{\prime} n_{i}^{\prime}$ for $i \in I_{\text {odd }}, s_{i}=1$ for $i \in I_{e v}$ with $n_{i}^{\prime} \in \mathbf{Z} / 2, \sum_{i \in I_{\text {odd }}} n_{i}^{\prime}=1$. We see that $\operatorname{ker} \zeta \subset A^{\prime}$. The opposite containment is obvious. This completes the proof when $I_{\text {odd }} \neq \emptyset$.

Assume next that $I_{\text {odd }}=\emptyset$. We have $\Delta=\left\{1, c_{*}\right\}$. In our case the homomorphism $\alpha$ in A.4(b) defines a connected covering of a simply connected group hence is an isomorphism; since $\Phi^{0}$ in A.4(b) is injective it follows that $\beta$ in A.4(b) is injective when restricted to $R$, hence $c \notin R$. We have $1=c^{n} \prod_{i \in I} \xi_{i}$. Hence $c^{n} \in R$. Since $c \notin R$ it follows that $n=0$. Thus, $1=\prod_{i \in I} \xi_{i}$. From this we deduce as in the proof of A.4(c) that $s=1$. This completes the proof of (a).

Let $\Delta^{+}$be the subgroup of $\Delta$ consisting of the elements of the form $c_{*}^{n} \prod_{i \in I_{\text {odd }}} z_{i}^{n_{i}}$ where $n_{i} \in \mathbf{Z} / 2, n \in \mathbf{Z} / 2, \sum_{i \in I_{\text {odd }}} n_{i}=0$. We have $\left|\Delta^{+}\right|=2^{\left|I_{\text {odd }}\right|}$ if $I_{\text {odd }} \neq \emptyset$, $\left|\Delta^{+}\right|=2$ if $I_{\text {odd }}=\emptyset$. We regard the semidirect product $S \cdot \Delta^{+}$as a subgroup of $S \cdot \Delta$ in an obvious way. Let $\zeta_{0}: S \cdot \Delta^{+} \rightarrow \mathcal{G}$ be the restriction of $\zeta: S \cdot \Delta \rightarrow \tilde{O}_{V}$. From (a) we deduce: 
(b) The kernel of $\zeta_{0}: S \cdot \Delta^{+} \rightarrow \mathcal{G}$ is equal to $A^{\prime}$.

The image of $\zeta_{0}$ is the subgroup of $\mathcal{G}$ generated by $R$, by $\tilde{y}_{i} \tilde{y}_{i^{\prime}}\left(i, i^{\prime} \in I_{\text {odd }}\right)$ and by $c$. Hence it is equal to $H$. We see that $\zeta_{0}$ defines an isomorphism

$$
\left(S \cdot \Delta^{+}\right) / A^{\prime} \stackrel{\sim}{\longrightarrow} H
$$

If $I_{\text {odd }} \neq \emptyset$, we can identify

$$
\left(S \cdot \Delta^{+}\right) / A^{\prime}=\left(S^{0} \cdot \Delta^{+}\right) / A_{1}^{\prime}
$$

where

$$
\begin{aligned}
& A_{1}^{\prime}=\left\{s c_{*}^{n} ; s \in S, n \in \mathbf{Z} / 2, s_{i}=c_{i}^{\prime n_{i}} \text { if } i \in I_{\text {odd }}^{\geq 2},\right. \\
& \left.s_{i}=1 \text { if } i \in I_{\text {even }} \cup\left(I_{\text {odd }}-I_{\text {odd }}^{\geq 2}\right), n_{i} \in \mathbf{Z} / 2, n=\sum_{i \in I_{\text {odd }}^{\geq 2}} n_{i}\right\} .
\end{aligned}
$$

We show:

(e) If $I_{\text {odd }}^{\geq 2}=\emptyset$ then $H=S^{0} \cdot \Delta^{+}$; hence $H / H^{0}=\Delta^{+}$.

Assume first that $I_{o d d}=\emptyset$. Then $A^{\prime}=\{1\}, \Delta^{+}=\left\{1, c_{*}\right\}, S=S^{0}$ and (c) becomes $S^{0} \sqcup S^{0} c_{*} \stackrel{\sim}{\longrightarrow} H$; thus (e) holds. If $I_{\text {odd }} \neq \emptyset$ and $I_{\text {odd }}^{\geq 2}=\emptyset$ then we have $A_{1}^{\prime}=\{1\}$ so that (e) follows from (d).

A.7. In this subsection we assume that $I_{\text {odd }}^{\geq 2} \neq \emptyset$. Using A.6(c),(d) we see that in this case we have $H^{0}=S^{0} / A_{2}^{\prime}$ where

$$
\begin{aligned}
& A_{2}^{\prime}=\left\{s \in S ; s_{i}=c_{i}^{\prime n_{i}} \text { if } i \in I_{\text {odd }}^{\geq 2}, s_{i}=1\right. \\
& \text { if } \left.i \in I_{\text {even }} \cup\left(I_{\text {odd }}-I_{\text {odd }}^{\geq 2}\right), n_{i} \in \mathbf{Z} / 2, \sum_{i \in I_{\text {odd }}^{\geq 2}} n_{i}=0\right\},
\end{aligned}
$$

so that for $i \in I_{\text {odd }}^{\geq 2}$, the image of $c_{i}^{\prime}$ in $H^{0}$ is independent of $i$; we denote it by $c^{\prime}$. Note that the $\Delta^{+}$action on $S^{0}$ induces an action of $\Delta^{+}$on $S^{0} / A_{2}^{\prime}$ and we can form the semidirect product $\left(S^{0} / A_{2}^{\prime}\right) \cdot \Delta^{+}$. From A.6(c),(d) we see that $H=\left(\left(S^{0} / A_{2}^{\prime}\right)\right.$. $\left.\Delta^{+}\right) /\left\{1, c^{\prime} c_{*}\right\}$. Now $\left(S^{0} / A_{2}^{\prime}\right)_{d e r}$ is the image of $\left(S^{0}\right)_{d e r}=\prod_{i \in I_{\text {even }} \cup I_{\text {odd }}^{\geq 3}} S_{i}$ under $p: S^{0} \rightarrow\left(S^{0} / A_{2}^{\prime}\right)_{d e r}$. The condition that $\left(S^{0} / A_{2}^{\prime}\right)_{d e r}$ is simply connected is that the restriction of $p$ to $\left(S^{0}\right)_{d e r}$ has trivial kernel; that kernel is

$$
\begin{aligned}
& A_{2}^{\prime} \cap\left(S^{0}\right)_{\text {der }}=\left\{s \in S ; s_{i}=c_{i}^{\prime n_{i}} \text { if } i \in I_{\text {odd }}^{\geq 3}, s_{i}=1\right. \\
& \text { if } \left.i \in I_{\text {even }} \cup\left(I_{\text {odd }}-I_{\text {odd }}^{\geq 3}\right), n_{i} \in \mathbf{Z} / 2, \sum_{i \in I_{\text {odd }}^{\geq 2}} n_{i}=0\right\},
\end{aligned}
$$

and this is trivial precisely when $\left|I_{\text {odd }}^{\geq 3}\right| \leq 1$. 
A.8. From the results in A.6,A.7 we see that $\left(H^{0}\right)_{\text {der }}$ is simply connected if and only if $\left|I_{\text {odd }}^{\geq 3}\right| \leq 1$.

A.9. Let $V$ be a $\mathbf{C}$-vector space of dimension $N \geq 4$ with a fixed symmetric nondegenerate symplectic form $():, V \times V \rightarrow \mathbf{C}$. Let $\mathcal{G}=I s_{V}$. We assume that $V=\oplus_{i \geq 1} V_{i}$ where $V_{i}=W_{i} \otimes E_{i}$ and $W_{i}, E_{i}$ are $\mathbf{C}$-vector spaces of dimension $i, m_{i}$ respectively with given nondegenerate bilinear forms $($,$) (so that if i$ is odd, $($,$) is symmetric for W_{i}$ and symplectic for $E_{i}$; if $i$ is even, (, ) is symmetric for $E_{i}$ and symplectic for $\left.W_{i}\right)$ such that $\left(w \otimes e, w^{\prime} \otimes e^{\prime}\right)=\left(w, w^{\prime}\right)\left(e, e^{\prime}\right)$ for $w, w^{\prime} \in W_{i}$, $e, e^{\prime} \in E_{i}$ and $\left(V_{i}, V_{j}\right)=0$ for $i \neq j$. Let $I=\left\{i \geq 1 ; m_{i} \geq 1\right\}, I_{o d d}=I \cap(2 \mathbf{Z}+1)$, $I_{\text {even }}=I \cap(2 \mathbf{Z})$.

We have an imbedding $\tau_{i}: I s_{E_{i}} \rightarrow I s_{V_{i}}, g \mapsto 1_{W_{i}} \otimes g$. Let $\Phi: \prod_{i \in I} I s_{E_{i}} \rightarrow \mathcal{G}$ be the homomorphism $\left(g_{i}\right) \mapsto \oplus_{i \in I} \tau_{i}\left(g_{i}\right)$ (an imbedding); it restricts to an imbedding $\Phi^{0}: \prod_{i \in I} I s_{E_{i}}^{0} \rightarrow \mathcal{G}$. Let $H=$ image of $\Phi$. Then $H$ is of the form $H(u)$ for some $u \in \mathcal{G}$ (as in 1.7) and all $H(u)$ as in 1.7 are obtained up to conjugacy. We have image of $\Phi^{0}=H^{0}$.

We see that $\left(H^{0}\right)_{\text {der }}$ is simply connected if and only if the number of $i \in I_{\text {even }}$ such that $m_{i} \geq 3$ is 0 .

A.10. In this subsection we assume that $L$ is the centralizer of a torus $S$ in $\mathcal{G}$, that $u \in L$ and that $\left(H^{0}\right)_{d e r}$ is simply connected. Let $\mathcal{V}^{\prime}$ be the unipotent radical of $Z_{L}(u)^{0}$; let $\mathcal{V}^{\prime \prime}$ be the unipotent radical of $Z_{L_{\text {der }}}(u)^{0}$. We show:

(a) $Z_{L}(u)=H^{\prime} \mathcal{V}^{\prime}$ (semidirect product) where $H^{\prime}$ is reductive and $\left(H^{\prime 0}\right)_{\text {der }}$ is simply connected;

(b) $Z_{L_{d e r}}(u)=H^{\prime \prime} \mathcal{V}^{\prime \prime}$ (semidirect product) where $H^{\prime \prime}$ is reductive and $\left(H^{\prime \prime 0}\right)_{\text {der }}$ is simply connected.

We can assume that $S \subset H$. We have $Z_{L}(u)=Z_{\mathcal{G}}(u) \cap Z_{\mathcal{G}}(S)=H V \cap Z_{\mathcal{G}}(S)=$ $Z_{H}(S) Z_{\mathcal{V}}(S)$. Thus we can assume that $H^{\prime}=Z_{H}(S)$ so that $H^{\prime 0}=Z_{H^{0}}(S)$. Since $\left(H^{0}\right)_{\text {der }}$ is simply connected, it follows that $\left(Z_{H^{0}}(S)\right)_{\text {der }}$ is simply connected, proving (a). Let $T$ be the connected centre of $L$. We have $L=L_{d e r} T$. Clearly, $Z_{L}(u)=Z_{L_{\text {der }}}(u) T$. Thus we can assume that $H^{\prime}=H^{\prime \prime} T$ so that $H^{\prime 0}, H^{\prime \prime 0}$ have the same derived group, proving (b).

A.11. In the remainder of this appendix we assume that $\mathcal{G}$ (in 1.7) is of exceptional type and $u, H(u)$ are as in 1.7. As G. Seitz pointed out to the author, the structure of $H=H(u)$ can in principle be extracted from [LS]. More precisely, the tables [LS, 22.3.1-22.3.5] contain information on the structure of the Lie algebra of $H^{0}$, on the structure of $H / H^{0}$ and the action of $H^{0}$ on the Lie algebra of $\mathcal{G}$. From this one can recover the precise structure of $H^{0}$; using in addition the tables [LS, 22.2.1-22.2.6] one can recover the structure of the extension $1 \rightarrow H^{0} \rightarrow H \rightarrow H / H^{0} \rightarrow 1$. (When $\mathcal{G}$ is of type $E_{7}$ or $E_{6}$ then, in addition to the corresponding tables in [LS] for the corresponding adjoint groups, we must use the tables for $E_{8}$ by regarding $\mathcal{G}$ as a derived subgroup of a Levi subgroup of a parabolic subgroup of a group of type $E_{8}$; we also use A.10.) In this way we find the following results.

If $\mathcal{G}$ is of type $E_{6}$ or $G_{2}$ then $\left(H^{0}\right)_{d e r}$ is simply connected. 
Assume now that $\mathcal{G}$ is of type $F_{4}$. If $u$ is of type $A_{1} \tilde{A}_{1}$ (notation of [LS]) then $H=H^{0}=P G L_{2}(\mathbf{C}) \times S L_{2}(\mathbf{C})$. If $u$ is of type $B_{3}$ (notation of [LS]) then $H=H^{0}=P G L_{2}(\mathbf{C})$. For all other $u,\left(H^{0}\right)_{d e r}$ is simply connected.

Assume now that $\mathcal{G}$ is of type $E_{7}$. If $u$ is of type $A_{2} A_{1}^{2}$ (notation of [LS]) then $H=H^{0}=S L_{2}(\mathbf{C})^{3} /\{ \pm 1\}$ with $\{ \pm 1\}$ imbedded diagonally in the centre of $S L_{2}(\mathbf{C})^{3}$. For all other $u,\left(H^{0}\right)_{\text {der }}$ is simply connected.

Assume now that $\mathcal{G}$ is of type $E_{8}$. If $u$ is of type $A_{2} A_{1}^{2}$ (notation of [LS]) then $H=H^{0}=\left(S L_{2}(\mathbf{C}) \times \operatorname{Spin}_{7}(\mathbf{C})\right) /\{ \pm 1\}$ with $\{ \pm 1\}$ imbedded diagonally in the centre of $S L_{2}(\mathbf{C}) \times \operatorname{Spin}_{7}(\mathbf{C})$. If $u$ is of type $A_{3} A_{2} A_{1}$ (notation of [LS]) then $H=H^{0}=P G L_{2}(\mathbf{C}) \times S L_{2}(\mathbf{C})$. If $u$ is of type $A_{4} A_{2}$ (notation of [LS]) then $H=$ $H^{0}=S L_{2}(\mathbf{C})^{2} /\{ \pm 1\}$ with $\{ \pm 1\}$ imbedded diagonally in the centre of $S L_{2}(\mathbf{C})^{2}$. If $u$ is of type $D_{4}\left(a_{1}\right) A_{2}$ (notation of [LS]) then $H=P G L_{3}(\mathbf{C}) \cdot \mathbf{Z} / 2$ (semidirect product with the generator of $\mathbf{Z} / 2$ acting on $P G L_{3}(\mathbf{C})$ by an outer involution). If $u$ is of type $D_{5}\left(a_{1}\right) A_{1}$ (notation of [LS]) then $H=H^{0}=P G L_{2}(\mathbf{C}) \times S L_{2}(\mathbf{C})$. If $u$ is of type $A_{6}$ (notation of [LS]) then $H=H^{0}=S L_{2}(\mathbf{C})^{2} /\{ \pm 1\}$ with $\{ \pm 1\}$ imbedded diagonally in the centre of $S L_{2}(\mathbf{C})^{2}$. For all other $u,\left(H^{0}\right)_{d e r}$ is simply connected.

\section{REFERENCES}

[KL] D.Kazhdan and G.Lusztig, Representations of Coxeter groups and Hecke algebras, Invent.Math. 53 (1979), 165-184.

[KmL] J.-L.Kim and G.Lusztig, Characters of unipotent representations of a semisimple p-adic group, arxiv:1208.0320.

[LS] M.W.Liebeck and G.M.Seitz, Unipotent and nilpotent classes in simple algebraic groups and Lie algebras, Math.Surveys and Monographs, vol. 180, Amer.Math.Soc., 2012.

[L1] G.Lusztig, Unipotent representations of a finite Chevalley group of type $E_{8}$, Quart.J.Math. 30 (1979), 315-338.

[L2] G.Lusztig, Characters of reductive groups over a finite field, Ann.Math.Studies, vol. 107, Princeton Univ.Press, Princeton, 1984.

[L3] G.Lusztig, Intersection cohomology complexes on a reductive group, Invent.Math. 75 (1984), 205-252.

[L4] G.Lusztig, Classification of unipotent representations of simple p-adic groups, Int. Math. Res. Notices (1995), 517-589.

[L5] G.Lusztig, Character sheaves on disconnected groups, I, Represent.Th. 7 (2003), 374-403.

[L6] G.Lusztig, Unipotent almost characters of simple p-adic groups, arxiv:1212.6540.

Department of Mathematics, M.I.T., Cambridge, MA 02139 